\title{
Classical nucleation theory of immersion freezing: sensitivity of contact angle schemes to thermodynamic and kinetic parameters
}

\author{
Luisa Ickes $^{1, a}$, André Welti ${ }^{1, b}$, and Ulrike Lohmann ${ }^{1}$ \\ ${ }^{1}$ Institute of Atmospheric and Climate Science, ETH Zurich, Zurich, Switzerland \\ ${ }^{a}$ now at: Department of Meteorology, Stockholm University, Stockholm, Sweden \\ bnow at: Leibniz Institute for Tropospheric Research, Leipzig, Germany \\ Correspondence to: Luisa Ickes (luisa.ickes@env.ethz.ch)
}

Received: 29 November 2015 - Published in Atmos. Chem. Phys. Discuss.: 18 January 2016

Revised: 19 July 2016 - Accepted: 12 December 2016 - Published: 3 February 2017

\begin{abstract}
Heterogeneous ice formation by immersion freezing in mixed-phase clouds can be parameterized in general circulation models (GCMs) by classical nucleation theory (CNT). CNT parameterization schemes describe immersion freezing as a stochastic process, including the properties of insoluble aerosol particles in the droplets. There are different ways to parameterize the properties of aerosol particles (i.e., contact angle schemes), which are compiled and tested in this paper. The goal of this study is to find a parameterization scheme for GCMs to describe immersion freezing with the ability to shift and adjust the slope of the freezing curve compared to homogeneous freezing to match experimental data.

We showed in a previous publication that the resulting freezing curves from CNT are very sensitive to unconstrained kinetic and thermodynamic parameters in the case of homogeneous freezing. Here we investigate how sensitive the outcome of a parameter estimation for contact angle schemes from experimental data is to unconstrained kinetic and thermodynamic parameters. We demonstrate that the parameters describing the contact angle schemes can mask the uncertainty in thermodynamic and kinetic parameters.

Different CNT formulations are fitted to an extensive immersion freezing dataset consisting of size-selected measurements as a function of temperature and time for different mineral dust types, namely kaolinite, illite, montmorillonite, microcline (K-feldspar), and Arizona test dust. We investigated how accurate different CNT formulations (with estimated fit parameters for different contact angle schemes) reproduce the measured freezing data, especially the time and particle size dependence of the freezing process. The results
\end{abstract}

are compared to a simplified deterministic freezing scheme. In this context, we evaluated which CNT-based parameterization scheme able to represent particle properties is the best choice to describe immersion freezing in a GCM.

\section{Introduction}

In mixed-phase clouds freezing of cloud droplets occurs by different pathways of heterogeneous freezing/nucleation. The nucleation process is initiated on the surface of an aerosol particle, called ice nucleus (IN), which either collides with a supercooled droplet (contact freezing), acts as cloud condensation nucleus ( $\mathrm{CCN}$ ) and causes freezing when the droplet is increasingly supercooled (immersion freezing), freezes immediately after $\mathrm{CCN}$ activation at supercooled conditions (condensation freezing), or provides a site where water vapor deposits as ice (deposition nucleation) (Vali, 1985).

In midlatitudes, where supercooled clouds are common, IN and their effect on precipitation formation through immersion freezing influence the hydrological cycle (Lohmann, 2002; Zeng et al., 2009; DeMott et al., 2010) and thereby, for example, the biosphere and agriculture. Aerosol particles determine the formation and ice-water ratio of mixed-phase clouds, thereby the cloud radiative properties and indirectly the radiation budget, which affects earth's climate. Therefore results of climate simulations in regional and global models are sensitive to the parameterization scheme used for heterogeneous ice formation and in particular immersion freezing as it is the most abundant freezing pathway (Ansmann et al., 
2009; Wiacek et al., 2010). One approach to parameterize immersion freezing in global and regional climate models is by classical nucleation theory (CNT), which requires approximations of the thermodynamics and kinetics of nucleation. Although computationally more expensive compared to empirical parameterization schemes, it allows a physical treatment of ice nucleation as function of temperature $T$, ice supersaturation $S_{\mathrm{i}}$, time $t$, and IN type (e.g., size, surface properties). Using a theoretical scheme has the advantage that the scheme is valid over the whole $T-S_{\mathrm{i}}$ space, which is mandatory for the use in a general circulation model (GCM), where all kinds of conditions occur (especially in certain regions like the Arctic, but also in simulations of future climate, where atmospheric conditions can be different from the present day or the pre-industrial epoch). Empirical schemes are often limited to the narrow conditions from which the scheme was derived and can lead to unphysical results when extrapolated. Therefore empirical schemes might not be representative for the future atmosphere or untypical atmospheric conditions. One example is the empirical Meyers et al. (1992) scheme, which was developed using measurements in midlatitudes and has been found to be inaccurate when extrapolated to Arctic conditions (Prenni et al., 2007).

Some parameters in the framework of CNT are so far unconstrained. At the same time results from the CNT are very sensitive to the choice of thermodynamic and kinetic parameters, in particular interfacial tension between ice and water $\sigma_{\text {iw }}$ and activation energy $\Delta g^{\#}$. Sensitivity of CNT on $\sigma_{\text {iw }}$ and $\Delta g^{\#}$ in the case of homogeneous freezing has been discussed in Ickes et al. (2015). Using CNT as an approach to parameterize immersion freezing in aerosol-climate models raises the question of the sensitivity of the parameterization scheme to $\sigma_{\text {iw }}$ and $\Delta g^{\#}$ in the case of heterogeneous freezing. Additionally there is a need to include and represent IN properties. We test three different schemes to describe the effect of an IN population on immersion freezing conditions and investigate the impact of the chosen scheme on the parameterization of immersion freezing. We also discuss strategies for how to evaluate the applicability of different CNT formulations.

The formalism of CNT for immersion freezing is summarized in Sect. 2. Advantages and disadvantages of certain formulations for the use in GCMs are discussed. In Sect. 3 the sensitivity of the immersion freezing nucleation rate $J_{\mathrm{imm}}\left(\mathrm{s}^{-1} \mathrm{~m}^{-2}\right)$ and the fit of the geometric term $f$ to thermodynamic and kinetic parameters is investigated by fitting and comparing the results to an ice nucleation measurement dataset of kaolinite (Welti et al., 2012). The section is followed by suggestions for how to evaluate the quality of a CNT parameterization scheme (Sect. 4). Section 5 presents CNT parameters estimated from experimental data for five different mineral dust types and in Sect. 5.1 the criteria from Sect. 4 are applied for three CNT formulations and compared to the performance of an empirical parameterization.
Throughout this paper we refer to "CNT formulations", indicating a specific CNT framework for immersion freezing based on Eq. (4), including parameterization schemes for $\sigma_{\text {iw }}, \Delta g^{\#}$, and the geometric term $f$ (contact angle scheme). CNT formulation No. 1 as an example is later on abbreviated by CNT1. We refer to "schemes" to discuss the different parameterization schemes for the geometric term $f$ or the contact angle $\alpha$ to express the ice nucleating surface properties of aerosol particles.

\section{Classical nucleation theory for immersion freezing}

In CNT freezing is described as a stochastic process by a temperature-dependent nucleation rate. In the case of homogeneous freezing (pure water droplets) statistical fluctuation of water molecules can lead to the formation of small ice-like structures (ice embryos) that lead to freezing of the supercooled droplet if they reach a certain critical size (ice germ). The nucleation rate describes the formation of ice germs leading to freezing over time. It consists of a thermodynamic and a kinetic component. The thermodynamic component describes the formation of ice embryos (determined by the thermodynamic energy barrier $\Delta G$ ) and the kinetic component describes the number of molecules, which can be incorporated into the ice embryo (determined by the activation energy barrier $\Delta g^{\#}$ ).

The presence of IN immersed in supercooled droplets facilitates ice nucleation compared to homogeneous nucleation by providing a catalytic surface. The IN surface reduces the thermodynamic energy barrier $\Delta G$ determined by $T, S_{\mathrm{i}}$, and $\sigma_{\text {iw }}$. The difference in nucleation with and without an IN, i.e., homogeneous or heterogeneous nucleation, is accounted for by the geometric term $f$, also called wetting factor, compatibility factor, or contact parameter. This term indicates the increased probability to nucleate a stable ice germ due to the presence of the IN surface because of the reduced number of water molecules necessary to form an ice germ. $f$ describes by how much the IN properties (of unknown nature) reduce the energy barrier for the formation of ice embryos on its surface compared to homogeneous freezing:

$$
\begin{aligned}
\Delta G & =f(\alpha) \times \Delta G_{\text {hom }} \\
& =f(\alpha) \times \frac{16 \pi}{3} \times \frac{v_{\text {ice }}^{2} \sigma_{\text {iw }}^{3}}{\left(k_{\mathrm{B}} T \ln S_{\mathrm{i}}\right)^{2}},
\end{aligned}
$$

where $v_{\text {ice }}$ is the volume of a water molecule in the ice embryo, $k_{\mathrm{B}}$ the Boltzmann constant, $S_{\mathrm{i}}$ the saturation ratio with respect to ice, $r_{\mathrm{IN}}$ the radius of the catalytic IN surface, and $r_{\text {germ }}$ the critical radius of an stable ice cluster which initiates freezing of the droplet.

$f$ can be expressed as a function of the contact angle $\alpha$, which is the tangential angle between the ice embryo on the IN surface and the parent phase (here supercooled water) (Fletcher, 1958): 


$$
\begin{aligned}
f(\alpha) & =\frac{1}{2}\left[1+\left(\frac{1-X \times \cos \alpha}{g}\right)^{3}\right. \\
& +X^{3}\left(2-3\left(\frac{X-\cos \alpha}{g}\right)+\left(\frac{X-\cos \alpha}{g}\right)^{3}\right) \\
& \left.+3 \times \cos \alpha \times X^{2}\left(\frac{X-\cos \alpha}{g}-1\right)\right]
\end{aligned}
$$$$
\text { with } X=\frac{r_{\mathrm{IN}}}{r_{\text {germ }}} \text { and } g=\sqrt{1+X^{2}-2 \times X \times \cos \alpha} \text {. }
$$

The contact angle $\alpha$ has a value between 0 and $180^{\circ}$, where the latter is equal to the case of homogeneous freezing ( $f=$ $1 \rightarrow \Delta G=\Delta G_{\text {hom }}$ ).

If the radius of the IN is significantly larger than the radius of the ice germ, curvature of the IN surface can be neglected leading to a simplified form of $f$ (Volmer, 1939):

$f(\alpha)=\frac{(2+\cos \alpha)(1-\cos \alpha)^{2}}{4}$.

Whereas the thermodynamic term in the nucleation rate $J_{\text {imm }}$ (thermodynamic exponent determined by the energy barrier $\Delta G$, see above) changes from homogeneous to heterogeneous freezing, the kinetic term is assumed to be the same for homogeneous and immersion freezing. The prefactor of the nucleation rate changes as well. It is defined as

$$
\begin{gathered}
C_{\text {prefac }}=n_{\mathrm{s}} \times 4 \pi r_{\text {germ }}^{2} \times Z \times \frac{k_{\mathrm{B}} T}{h} \times N_{\mathrm{l}}, \\
\text { with } Z=\frac{1}{n_{k, \text { germ }}} \times \sqrt{\frac{\Delta G}{3 \pi k_{\mathrm{B}} T},}
\end{gathered}
$$

where $n_{\mathrm{s}}$ is the number of water molecules in contact with the unit area of the ice cluster, $Z$ the non-equilibrium Zel'dovich factor, $h$ the Planck's constant, and $N_{1}$ the volume-based number density of water molecules in the liquid parent phase. $Z$ is not the same for homogeneous and heterogeneous freezing because the number of the water molecules in the ice germ, $n_{k, \text { germ }}$, differs. As shown in Pruppacher and Klett (2000), most of the prefactors cancel out in the case of heterogeneous freezing leading to the following expression for the nucleation rate for immersion freezing:

$$
\begin{aligned}
J_{\mathrm{imm}}\left(\mathrm{m}^{-2} \times \mathrm{s}^{-1}\right)=n_{\mathrm{s}} \times \frac{k_{\mathrm{B}} T}{h} \\
\quad \times \underbrace{\exp \left(-\frac{\Delta g^{\#}}{k_{\mathrm{B}} T}\right)}_{\text {kinetic comp. }} \times \underbrace{\exp \left(-\frac{f(\alpha) \times \Delta G_{\mathrm{hom}}}{k_{\mathrm{B}} T}\right)}_{\text {thermodyn. comp. }} .
\end{aligned}
$$

Since the energy barrier of immersion freezing is reduced compared to homogeneous freezing, $J_{\text {imm }}$ is higher compared to the homogeneous nucleation rate $J_{\text {hom }}$ and exhibits a different $T$ dependence (less steep at a certain $T$ ). The shift of the freezing curve is described by the geometric term $f$ and has to be captured by the different CNT formulations.

\subsection{Parameterization schemes for the geometric term $f$}

Different parameterization schemes for $f$ have been put forward to describe the influence of an IN on the nucleation process, i.e., to describe the ice nucleating surface properties of aerosol particles, e.g., Marcolli et al. (2007). Depending on the scheme the IN properties are represented by functions with one or several fit parameters and the complexity for an implementation in a GCM differs accordingly. Note that increasing complexity comes with higher computational costs (Hurrell et al., 2009). Based on the computational complexity we chose three schemes, including one or two fit parameters for the following sensitivity analysis (Sect. 3). They are briefly explained here followed by a paragraph about computational costs. A graphical representation of each scheme is shown in Fig. 1. For more details see Marcolli et al. (2007) and Lüönd et al. (2010).

From immersion freezing measurements the frozen fraction $F F$ is obtained, which is the fraction of a droplet population that is frozen at a certain temperature $T$ after a certain time $t$. To compare CNT-based parameterization schemes to measurements, $F F$ is calculated from the nucleation rate $J_{\text {imm. }}$. The frozen fraction $F F$ is given by

$F F=1-\exp \left(-J_{\mathrm{imm}}(T, \alpha) \times A_{\mathrm{IN}} \times \Delta t\right)$,

with $A_{\text {IN }}$ being the surface area of the IN. For simplicity particles are assumed to be spherical $\left(A_{\mathrm{IN}}=4 \pi r_{\mathrm{IN}}^{2}\right)$. Thus, the surface used for the IN of a specific mass represents a lower limit (non-spherical particles would be larger).

\subsubsection{Single- $\alpha$ scheme}

The single- $\alpha$ scheme assigns one contact angle to the entire surface of all particles. It is based on the assumption that all particles have one uniform surface property responsible for their ice nucleating ability. Consequently all particles have an equal probability to act as IN at given conditions. The scheme requires only one fit parameter $(f$ or $\alpha$ ). One variation of the single- $\alpha$ scheme, the single- $\alpha+$ fit $\Delta g^{\#}$ scheme (see Sect. 3), requires two fit parameters ( $f$ or $\alpha$ and $\Delta g^{\#}$ ).

It is the least complex and consequently the cheapest scheme suitable for implementation in GCMs. However, it does not take into account that ice nucleating properties might be variable throughout a particle population. This scheme is used in several models, e.g., Khvorostyanov and Curry (2000, 2004, 2005), Liu et al. (2007), Eidhammer et al. (2009), Hoose et al. (2010), Storelvmo et al. (2011), and Ervens and Feingold (2012).

\subsection{2 $\alpha$-pdf scheme}

The $\alpha$-pdf scheme is an extension of the single- $\alpha$ scheme. It assumes a heterogeneity of particles in an aerosol population by using a log-normal probability density function (pdf) for the contact angle $\alpha p(\alpha)$. The log-normal distribution of $\alpha$ 


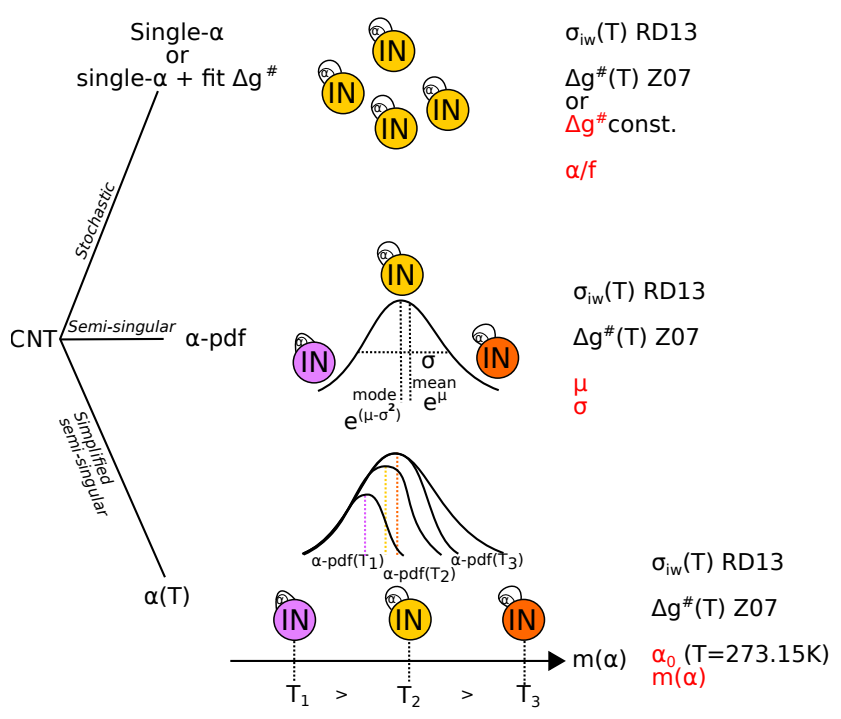

Figure 1. Schematic sketch of different contact angle schemes in which the fit parameters for each contact angle scheme are marked in red. Stochastic: all IN have the same contact angle $\alpha$ (single$\alpha$ ). The ice germ with the contact angle $\alpha$ is shown in the sketch. Semi-singular: different IN have different contact angles. The contact angles are distributed with an $\alpha$-pdf over the IN population. The sketch shows three contact angles $\alpha_{1}$ (purple), $\alpha_{2}$ (yellow), and $\alpha_{3}$ (orange) of the contact angle distribution. The most efficient contact angle $\alpha_{1}$ is the smallest ( $<\alpha_{2}<\alpha_{3}$ ). Simplified semi-singular: all IN have the same contact angle. The contact angle changes with $T$ ( $\alpha_{1}<\alpha_{2}<\alpha_{3}$ in the example). $\alpha$ is equivalent to the mean contact angle of the $\alpha$-pdf scheme ( $\mathrm{e}^{\mu}$ ) or mean activity of the $\alpha$-pdf distribution, which changes with temperature. Figure adapted from F. Lüönd.

within a particle population is expressed by two fit parameters, the logarithmic mean contact angle $\mu$, and the variance $\sigma$ of the distribution:

$p(\alpha)=\frac{1}{\alpha \sqrt{2 \pi \sigma^{2}}} \times \exp \left(-\frac{(\ln (\alpha)-\mu)^{2}}{2 \sigma^{2}}\right)$.

This approach attributes an individual surface property to each particle on the entire particle surface.

The variance $\sigma$ accounts for the heterogeneity of the particle property within the aerosol population: the larger the variance $\sigma$, the larger the heterogeneity among the particles. The frozen fraction $F F$ is derived by integrating the contact angle distribution over all possible contact angles:

$$
F F=1-\int_{0}^{\pi} p(\alpha) \times \exp \left(-J_{\mathrm{imm}}(T, \alpha) \times A_{\mathrm{IN}} \times \Delta t\right) \mathrm{d} \alpha .
$$

The approach has been frequently used to interpret freezing data, e.g., Marcolli et al. (2007), Lüönd et al. (2010), Broadley et al. (2012), Welti et al. (2012), and Wheeler et al. (2014). Due to the increased complexity compared to the single- $\alpha$ scheme, only a few attempts have been made to implement it in GCMs (e.g., Wang et al., 2014). Application of the scheme in GCMs creates the problem of unknown time evolution of the contact angle distribution. Because the most efficient IN will form ice first, the remaining contact angle distribution (IN, which did not freeze yet) changes in case an aerosol population is not replenished within one timestep. Without an explicit treatment of the time evolution of the contact angle distribution, ice formation will be overestimated since the most efficient IN can initiate freezing over and over again. The time evolution of the contact angle distribution can be neglected by assuming that the aerosol particles are replenished within one model timestep. Note that this issue is closely connected to the time resolution of the GCM, which will be discussed in a future publication.

Another extension of the single- $\alpha$ and frequently used scheme is the active sites scheme, e.g., in Marcolli et al. (2007), Lüönd et al. (2010), Niedermeier et al. (2011), Welti et al. (2012), and Wheeler et al. (2014). It goes one step further and assumes several surface sites with different contact angle on a single IN. In this case the active sites have to be memorized over several timesteps, which means that at least one extra tracer is needed in the model. This makes this scheme computationally too expensive for the use in GCMs (see Sect. 2.1.4). Thus, it is left out in the following analysis. More information about the active sites scheme can be found in Marcolli et al. (2007), Lüönd et al. (2010), and Niedermeier et al. (2011).

\subsubsection{Temperature-dependent single- $\alpha$ scheme $(\alpha(T)$ scheme)}

The $\alpha(T)$ scheme is a compromise between the single- $\alpha$ and the $\alpha$-pdf scheme. It assumes that $\alpha$ is different for different $T$, which reflects a change of the activated fraction of the $\alpha$-pdf distribution and with that a change in apparent contact angle. Efficient IN (with small contact angles) freeze at highest temperatures. The lower the temperature is, the higher the chance that particles with larger contact angles can be activated. This leads to a change of the apparent contact angle to larger $\alpha$ with further cooling due to the shift of the activated fraction of the $\alpha$-pdf distribution with supercooling. The $\alpha(T)$ scheme thus represents the shifted mean contact angle of an initial contact angle distribution but does not take into account how contact angles are distributed among a particle population at a certain temperature. The temperature dependence of $\alpha$ can be approximated to be linear as discussed in Welti et al. (2012). Being capable to describe a variability of the freezing process due to a contact angle distribution without being computationally complex makes the $\alpha(T)$ scheme attractive for GCMs. However, it demands an indirect assumption on how the aerosol population changes with supercooling. It does not circumvent the issue of shifting the contact angle distribution with time. 
The frozen fraction $F F$ is estimated analogously to the single- $\alpha$ scheme using a linear function for $\alpha(T)$ :

$$
\begin{gathered}
F F=1-\exp \left(-J_{\mathrm{imm}}(T, \alpha(T)) \times A_{\mathrm{IN}} \times \Delta t\right), \\
\text { with } \alpha(T)=\alpha_{0}+m \times(T-273.15 \mathrm{~K}) .
\end{gathered}
$$

Note that in general the temperature dependence of $\alpha$ can be interpreted either as a result of the temperature dependence of the interfacial tensions ( $\sigma_{\text {is }}$ and $\sigma_{\text {iw }}$ ) or as the apparent contact angle of an ensemble with a diversity of contact angles from particle to particle. In contrast to Zobrist et al. (2007), Alpert et al. (2011), Knopf and Forrester (2011), and Rigg et al. (2013), we follow the second interpretation (simplified temperature-dependent $\alpha$-pdf scheme) as explained above. Accounting for a physical dependence of $\alpha$ on $T$ as a result of the temperature dependence of the interfacial tensions leads to a decrease of $\alpha$, which is contradictory to the assumption we made here.

\subsubsection{Computational costs}

Since computational costs are strongly linked to the complexity of parameterization schemes (Hurrell et al., 2009), the complexity of a parameterization scheme is an important factor which has to be considered before implementation into a GCM. A general quantification of the computational costs of parameterization schemes depends an many aspects (treatment of aerosol particles and cloud microphysics) of the GCM used. The number and kind of variables needed, e.g., $T$, size, and number of a certain aerosol type, to derive $J_{\text {imm }}$ is an indicator for the complexity of the parameterization scheme. As soon as a variable needs to be memorized over several timesteps, e.g., change in contact angle distribution, an extra-tracer is required, which might be computationally expensive.

The cheapest CNT-based parameterization schemes are the single- $\alpha$ scheme and the $\alpha(T)$ scheme. The $\alpha$-pdf scheme is computationally more expensive because the contact angle distribution changes with time if the contact angles are not replenished from one timestep to the next. An explicit treatment of time evolution of the contact angle distribution requires extra tracers to memorize which contact angles from the distribution were already used in the timesteps before and thus make the scheme computationally more expensive. Using extra tracers for the contact angle of mineral dust particles would approximately lead to an increase of computational costs of $21 \%$ in the GCM ECHAM6-HAM2. If the time evolution of the contact angle distribution is not taken into account, the $\alpha$-pdf scheme becomes computationally similarly expensive as the single- $\alpha$ and the $\alpha(T)$ scheme. However, the integral in Eq. (7) cannot be solved analytically. Therefore, to minimize computational costs, a lookup table could be used instead of discretized finite sums. Using lookup tables is dependent on the size and format of the lookup table more expensive compared to solving an equation with simple constants as in the case of the single- $\alpha$ and
$\alpha(T)$ scheme. The $\alpha(T)$ scheme is a simplified version of the $\alpha$-pdf and computationally cheaper because no integration over a contact angle distribution is necessary. The active sites scheme requires extra tracers as well, which would lead to high computational costs (comparable to the $\alpha$-pdf with explicit change of contact angle distribution with time).

\section{Sensitivity analysis}

\subsection{Fitting immersion freezing measurements}

In this section, the sensitivity of $J_{\text {imm }}$ and $F F$ to different combinations of $\sigma_{\mathrm{iw}}$ and $\Delta g^{\#}$ (see Ickes et al., 2015, for a discussion of these parameters) in combination with the contact angle schemes discussed in Sect. 2.1 is analyzed by fitting and comparing the different CNT parameterization schemes to experimental data. This helps us to understand how fit parameters influence the calculated freezing curves.

The experimental data taken from Welti et al. (2012) consist of optically detected frozen fractions $F F$ of droplets containing single immersed, monodisperse kaolinite (Fluka) particles. The $F F$ was measured as a function of $T$, the particle radius $r_{\mathrm{IN}}$, and the residence time in the measurement setup $t$. Experiments were performed using IMCA/ZINC (see Welti et al., 2012, for details). The error bars of the data reflect the uncertainty in the distinction of water droplets and ice crystals in the detection unit. For the sensitivity analysis the dataset measured after $10 \mathrm{~s}$ for kaolinite particles with a diameter of $400 \mathrm{~nm}$ is used. Note that the size of the particles might be underestimated due to the assumption of sphericity and therefore the calculated nucleation rates $J_{\text {imm }}$ from experimental frozen fractions represent a lower limit.

To explore the sensitivity of $J_{\text {imm }}$ to thermodynamic and kinetic parameters we use different CNT formulations with thermodynamic and kinetic parameters from different studies. The focus is on $\sigma_{\text {iw }}$. In the following all CNT formulations, which are used for the analysis, are listed. An overview is given in Table 1. Capital letters in the naming indicate the author from whose publication thermodynamic and kinetic parameters are obtained.

\section{- No. 1: single- $\alpha$ R\&D $+Z$}

The first approach is to use a single- $\alpha$ scheme in combination with the thermodynamic and kinetic parameters shown to be in good agreement with homogeneous nucleation rates (see Ickes et al., 2015). When using a single- $\alpha$ scheme it might be important that the kinetic and thermodynamic parameters are a combination which reproduces the homogeneous freezing data well as there is only one fit parameter and deviations cannot be compensated by additional parameters. The emerged best fitting combination of $\sigma_{\text {iw }}$ and $\Delta g^{\#}$ from the analysis of homogeneous data is $\sigma_{\text {iw }}$ from Reinhardt and Doye (2013) and $\Delta g^{\#}$ from Zobrist et al. (2007). We 
Table 1. Overview of CNT formulations used for the sensitivity analysis, results for the fit parameters, and evaluation of the fit result. The values are rounded to two digits after the decimal point. The logarithmic mean contact angle $\mu$ and the temperature-dependent contact angles are converted into the corresponding geometric term $f$ using Eq. (3). CNT formulation nos. 1, 5, and 7 use the best fitting combination of $\sigma_{\mathrm{iw}}$ and $\Delta g^{\#}$ emerging from the homogeneous freezing analysis in Ickes et al. (2015).

\begin{tabular}{|c|c|c|c|c|c|}
\hline No. & Parameterization for $\sigma_{\mathrm{iw}}$ and $\Delta g^{\#}$ & $\begin{array}{l}\text { Contact angle } \\
\text { scheme }\end{array}$ & Name & Fit parameters & RMSE \\
\hline 1 & $\begin{array}{l}\sigma_{\text {iw }}: \text { Reinhardt and Doye }(2013) \\
\Delta g^{\#}: \text { Zobrist et al. }(2007) \approx 5 \times 10^{-20} \mathrm{~J}\end{array}$ & single- $\alpha$ & single- $\alpha \mathrm{R} \& \mathrm{D}+\mathrm{Z}$ & $1: f=0.55$ & 0.12 \\
\hline 2 & $\begin{array}{l}\sigma_{\mathrm{iw}}: \text { Reinhardt and Doye (2013) } \\
\Delta g^{\#}: \text { constant }\end{array}$ & single- $\alpha$ & single- $\alpha \mathrm{R} \& \mathrm{D}+$ fit $\Delta g^{\#}$ & $\begin{array}{l}2: f=0.24 \\
\Delta g^{\#}=11.01 \times 10^{-20} \mathbf{J}\end{array}$ & 0.05 \\
\hline 3 & $\begin{array}{l}\sigma_{\mathrm{iw}} \text { from Ouchi }(1954) \\
\Delta g^{\#}: \text { constant }\end{array}$ & single- $\alpha$ & single- $\alpha \mathrm{O}+$ fit $\Delta g^{\#}$ & $\begin{array}{l}2: f=0.69 \\
\Delta g^{\#}=12.3 \times 10^{-20} \mathrm{~J}\end{array}$ & 0.05 \\
\hline 4 & $\begin{array}{l}\sigma_{\text {iw }} \text { from Eadie }(1971) \\
\Delta g^{\#}: \text { constant }\end{array}$ & single- $\alpha$ & single- $\alpha \mathrm{E}+$ fit $\Delta g^{\#}$ & $\begin{array}{l}2: f=0.23 \\
\Delta g^{\#}=10.46 \times 10^{-20} \mathrm{~J}\end{array}$ & 0.05 \\
\hline $2 / 4$ & $\begin{array}{l}\sigma_{\text {iw }}: \text { Reinhardt and Doye (2013) } \\
\Delta g^{\#}: \text { constant }\end{array}$ & single- $\alpha$ & single- $\alpha \mathrm{R} \& \mathrm{D}+$ fit $\Delta g^{\#}(\mathrm{E})$ & $\begin{array}{l}2: f=0.24(\text { No. } 2) \\
\Delta g^{\#}=10.46 \times 10^{-20} \text { J (No. 4) }\end{array}$ & 0.31 \\
\hline 5 & same as No. 1 & $\alpha$-pdf & $\alpha$-pdf R\&D + Z & $\begin{array}{l}2: \mu=0.5 \\
\Rightarrow \alpha_{\text {mean }}=94.46^{\circ} \\
\hat{=} f_{\text {mean }} \approx 0.56 \\
\sigma=0.04\end{array}$ & 0.05 \\
\hline 6 & $\begin{array}{l}\sigma_{\mathrm{iw}} \text { from Eadie (1971) } \\
\Delta g^{\#}: \text { Zobrist et al. }(2007)\end{array}$ & $\alpha$-pdf & $\alpha$-pdf E $+Z$ & $\begin{array}{l}2: \mu=0.44 \\
\Rightarrow \alpha_{\text {mean }}=88.96^{\circ} \\
\hat{=} f_{\text {mean }} \approx 0.48 \\
\sigma=0.03\end{array}$ & 0.05 \\
\hline 7 & same as No. 1 & $\alpha(T)$ & $\alpha(T) \mathrm{R} \& \mathrm{D}+\mathrm{Z}$ & $\begin{array}{l}2: \alpha_{0}=0.7 \mathrm{rad}=40.1^{\circ} \\
\hat{=} f_{260} \approx 0.18 \\
f_{250} \approx 0.37 \\
f_{240} \approx 0.59 \\
m=-0.03 \mathrm{rad} \mathrm{K}^{-1}\end{array}$ & 0.05 \\
\hline
\end{tabular}

tested whether these formulations of $\sigma_{\mathrm{iw}}$ and $\Delta g^{\#}$ are also applicable to reproduce heterogeneous nucleation rates in combination with the single- $\alpha$ scheme.

- No. 2: single- $\alpha$ R\&D + fit $\Delta g^{\#}$

A second approach, which emerged from Chen et al. (2008), is using a constant $\Delta g^{\#}$ as an additional fit parameter to $f / \alpha$ instead of taking a temperaturedependent formulation. This assumption might be wrong in the context of homogeneous freezing especially at very low $T$ (Barahona, 2015). However, it should be applicable for immersion freezing conditions as the change in $\Delta g^{\#}$ is small in the corresponding temperature range. The approach is used in combination with $\sigma_{\text {iw }}$ from Reinhardt and Doye (2013) and a single$\alpha$ scheme. To decide whether $\sigma_{\text {iw }}$ from Reinhardt and Doye (2013) is the best choice, different expressions for $\sigma_{\text {iw }}$ are tested against a fit of the homogeneous nucleation rate $J_{\text {hom }}$ using constant $\Delta g^{\#}$ (see Fig. 2 analogous to Fig. 17 in Ickes et al., 2015). We find that $\sigma_{\text {iw }}$ from Reinhardt and Doye (2013) remains an appropriate choice even when $\Delta g^{\#}$ is used as a constant.

- Nos. 3 and 4: single- $\alpha \mathrm{O}+$ fit $\Delta g^{\#}$ and single- $\alpha \mathrm{E}+$ fit $\Delta g^{\#}$
To capture the whole possible range, two formulations of $\sigma_{\text {iw }}$ are used: one from Eadie (1971), leading to the lowest homogeneous nucleation rate, and a second formulation of $\sigma_{\text {iw }}$ from Ouchi (1954), leading to the highest homogeneous nucleation rate (see Fig. 2). For a summary of the two formulations of $\sigma_{\text {iw }}$ we refer to Ickes et al. (2015).

These two extremes of $\sigma_{\text {iw }}$ are used together with a constant $\Delta g^{\#}$ (fit parameter) and the single- $\alpha$ scheme to clarify whether a fit of $\alpha$ can compensate for a low/high $\sigma_{\text {iw }}$.

\section{- No. 5: $\alpha$-pdf R\&D + Z}

This CNT formulation is an $\alpha$-pdf scheme using the same thermodynamic and kinetic parameter as the CNT formulation for the single- $\alpha$ scheme (CNT1). The $\alpha$-pdf increases the complexity and adds an additional fit parameter compared to the single- $\alpha \mathrm{R} \& \mathrm{D}+\mathrm{Z}$ formulation (No. 1). Thus we test the influence of the choice of the contact angle scheme on the fit result. Additionally we examine whether the number of free fit parameters has an impact on the result.

- No. 6: $\alpha$-pdf E+Z 


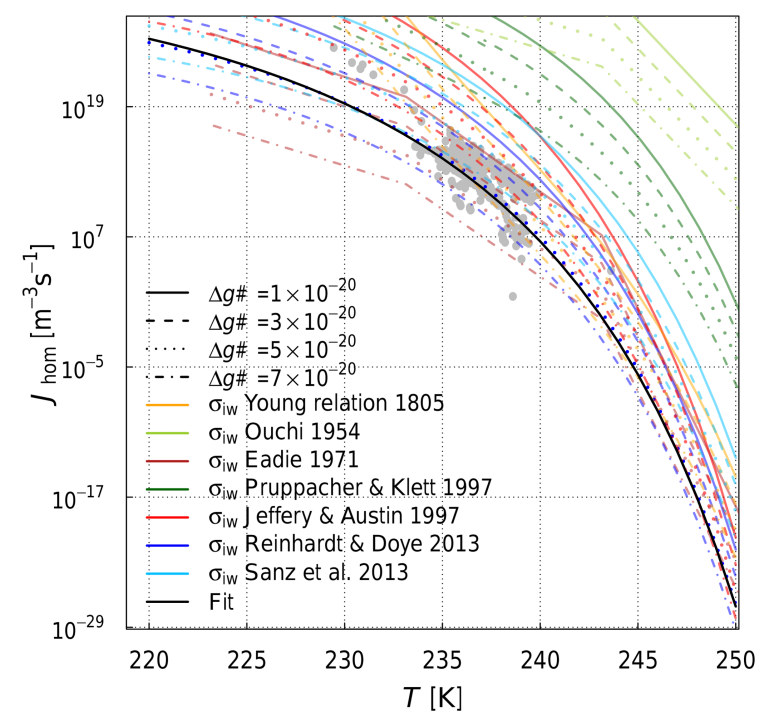

Figure 2. Comparison of the fitted $J_{\text {hom }}(T)$ (solid black line) with calculated nucleation rates using different formulations of $\sigma_{\text {iw }}$ and constant values for $\Delta g^{\#}$. Grey dots show experimental homogeneous freezing data. $\sigma_{\text {iw }}$ from Reinhardt and Doye (2013) captures the homogeneous freezing curve best.

One extreme formulation of $\sigma_{\mathrm{iw}}$ from Eadie (1971) is used together with $\Delta g^{\#}$ from Zobrist et al. (2007) and the $\alpha$-pdf scheme (see No. 5.).

\section{- No. 7: $\alpha(T)$ R\&D + Z}

Similar to the $\alpha$-pdf R\&D+Z formulation (CNT5), thermodynamic and kinetic parameter from the formulation for the single- $\alpha$ scheme (CNT1) are used with an $\alpha(T)$ scheme instead of the single- $\alpha$ scheme.

The dataset from Welti et al. (2012) is fitted with the previously listed CNT formulations. The fits are done by leastsquare minimization of $F F$ as a function of $T$. The results are shown in Fig. 3 and Table 1. Table 1 additionally contains information on $\Delta g^{\#}$ and $\sigma_{\mathrm{iw}}$. Overall most CNT formulations with two fit parameters are able to capture the freezing data well with similar root mean square errors (RMSE) of the estimated freezing curve and measured freezing data independently of the thermodynamic and kinetic parameters. In the following the results are discussed in more detail.

The single- $\alpha \mathrm{R} \& \mathrm{D}+\mathrm{Z}$ formulation (CNT1) poorly captures the experimental data and results in a too steep freezing curve. With the single- $\alpha$ scheme it is not possible to reproduce the reduction of the energy barrier in a correct manner and to decrease the temperature dependence of the nucleation rate from the homogeneous to the heterogeneous case. Having only one fit parameter, which in this case is a factor in the exponential term, is not sufficient to shift and flatten the freezing curve compared to homogeneous freezing. Only the $T$-shift of the freezing curve compared to homogeneous freezing is captured by the fitted single- $\alpha$ scheme.

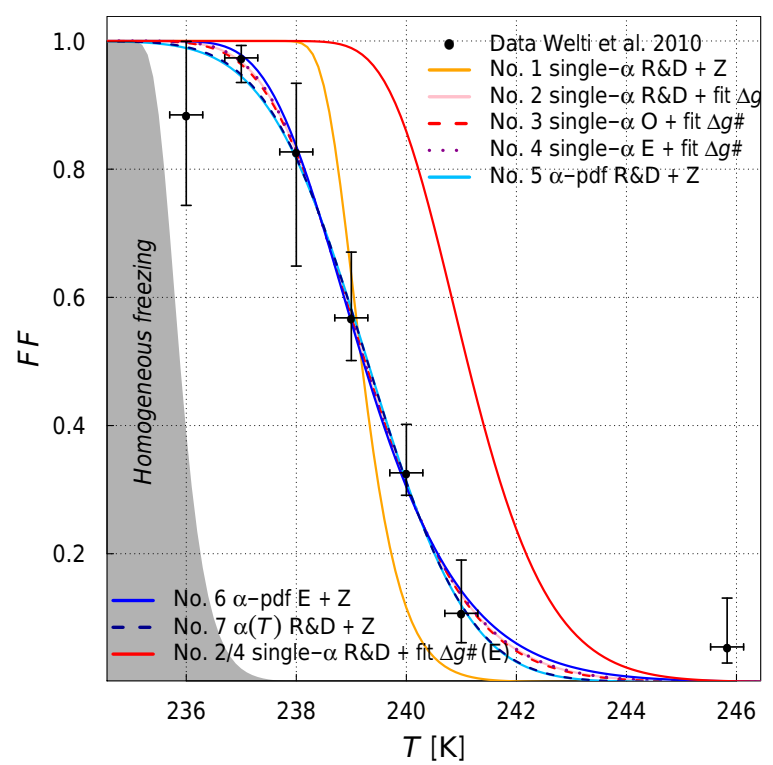

Figure 3. Calculated frozen fraction $F F$ as a function of $T$ for different thermodynamic and kinetic parameters in combination with different contact angle schemes for kaolinite with a particle diameter of $400 \mathrm{~nm}$ after a residence time of $10 \mathrm{~s}$. More details can be found in Table 1.

This can be seen in a more general illustration in Figs. A1 and A2 (Appendix A). This result indicates that the simplified assumption of only reducing $\Delta G$ by a single contact angle for the entire population is not sufficient. However, early experiments on $\mathrm{AgI}$ and recent molecular dynamics simulations (e.g., Cabriolu and Li, 2015) support the applicability of single- $\alpha$ in certain cases. Possibly a single- $\alpha$ scheme can be used for highly efficient IN triggering ice formation at low supercooling. In the context of the Young equation (Young, 1805 ) the assumption of a single- $\alpha$ scheme is questionable. If $\alpha$ represents the balance of the three surface tensions it has to change with $T$ since the surface tensions are temperature dependent.

Using $\Delta g^{\#}$ as an additional fit parameter (CNT2) can flatten the curve. In this case both fit parameters are factors in the exponential term of the nucleation rate with a similar influence on the fitted $F F$. The fit parameter which is multiplied with the temperature-dependent variable $\Delta G(f)$ mainly shifts the freezing curve but cannot reduce the steepness sufficiently at the same time (see single- $\alpha$ scheme). Using a second fit parameter $\Delta g^{\#}$ resolves this issue. A simplified view on this is that one fit parameter is responsible for the shift and the other one for the flattening of the immersion freezing curve compared to homogeneous freezing. Using a constant $\Delta g^{\#}$ might be reasonable based on the results from the homogeneous freezing analysis (Ickes et al., 2015), but fitting $\Delta g^{\#}$ to immersion freezing data leads to substantially higher $\Delta g^{\#}$ than those estimated by theoretical calculations (see Ickes et al., 2015). Moreover the fit value of $\Delta g^{\#}$ 
is aerosol specific. This might be an artificial result and it is questionable if the assumption of a temperature-independent and aerosol-type-specific (due to the fitting) $\Delta g^{\#}$ is a physical valid approach. It contradicts the assumption that the kinetic parameters such as $\Delta g^{\#}$ are the same for homogeneous and heterogeneous nucleation. The general approach to take the same thermodynamic and kinetic parameters (besides $f$ and the prefactor) for homogeneous and heterogeneous nucleation is based on the assumption that mechanisms (e.g., the diffusion of water molecules across the water-ice boundary) in the supercooled water are not influenced by the immersed aerosol particle. This hypothesis might not be true. The aerosol particle might influence, e.g., the diffusion of water molecules close to the particle, which could justify a change in $\Delta g^{\#}$ depending on aerosol type. A disturbance of the diffusion by an aerosol particle could, for example, be related to an impact on the hydrogen bond network by IN surface charges or polarizability of the particle surface (Edwards and Evans, 1962).

An alternative to having $\Delta g^{\#}$ as an additional fit parameter is to use a more sophisticated contact angle scheme, e.g., the $\alpha$-pdf scheme (CNT5 and CNT6) or the $\alpha(T)$ scheme (CNT7). Both approaches lead to good fits of the experimental freezing data and can be physically justified because they resemble the natural variability of an IN population. The $\alpha(T)$ scheme has the disadvantage that it is not inherently known how the apparent $\alpha$ changes with $T$. A wrong assumption could lead to an unphysical contact angle scheme.

The curves resulting from CNT 3-7 (single- $\alpha+$ fit $\Delta g^{\#}$ schemes, $\alpha$-pdf schemes, and $\alpha(T)$ scheme) all support the hypothesis that increasing the number of fit parameters from one to two allows to find a reasonable fit, independent of the kinetic and thermodynamic parameters chosen and also independently of the contact angle scheme. This is also supported by the result of the single- $\alpha$ scheme, where one fit parameter alone cannot shift and flatten the freezing curve. However, using a single- $\alpha$ scheme with a different additional fit parameter (e.g., the slope of $\sigma_{\text {iw }}$ instead of a constant $\Delta g^{\#}$ ) does not lead to a better fit of the freezing curve. This might be due to the formula of the energy barrier preventing a sufficiently large influence of the additional fit parameter on the steepness of the curve. $\Delta g^{\#}$ as an additional fit parameter is able to reduce the steepness of the freezing curve as it has the opposite temperature dependence compared to the energy barrier $\Delta G$. For a visualization of how the fit parameters influence the freezing curve for each scheme, please see Figs. A1 and A2 (Appendix A).

Using one CNT formulation, e.g., the single- $\alpha$ R\&D + fit $\Delta g^{\#}$ formulation (CNT2), together with fit parameters, e.g., $\alpha$, emerging from a fit with a different CNT formulation, e.g., the single- $\alpha \mathrm{E}+$ fit $\Delta g^{\#}$ formulation (CNT4), leads to a wrong freezing curve, which is illustrated in Fig. 3 (solid red line, single- $\alpha \mathrm{R} \& \mathrm{D}+$ fit $\Delta g^{\#}(\mathrm{E}) ;$ No. $\left.2 / 4\right)$. It is an example of the implication if fit parameters were used together with a different CNT formulation to the one used to derive the fit parameters. This can unintentionally happen in GCMs if an implemented CNT formulation were later extended, e.g., by another aerosol species, and no care is taken to ensure that the fit parameters for the new species from the literature are derived from the same CNT formulation as the one implemented in the model.

Looking at Table 1 one can see that $f$ differs substantially, e.g., when using the single- $\alpha+$ fit $\Delta g^{\#}$ scheme with different values for $\sigma_{\text {iw }}$. Comparing $\sigma_{\text {iw }}$ from Reinhardt and Doye (2013) with $\sigma_{\text {iw }}$ from Ouchi (1954) leads to a difference in fitted $f$ of more than $300 \%$, which translates into a difference in contact angle $\alpha$ of approximately $75^{\circ}$. However, all single- $\alpha+$ fit $\Delta g^{\#}$ schemes result in a nearly similar freezing curve with the same RMSE. The fit parameters from the contact angle scheme compensate inaccuracies associated with from thermodynamic and kinetic parameters and thus mask potentially wrong assumptions, e.g., $\sigma_{\mathrm{iw}}$ of Ouchi (1954). This makes it challenging to compare contact angles or fit parameters from different studies when the same CNT formulation was not used. Hence in the next subsection we investigate how fit results vary when thermodynamic and kinetic parameters differ and whether there is possible to compare fit parameters from different studies using different CNT formulations.

\subsection{Uncertainty of fitting $\alpha$ and $\alpha$-pdf}

Table 1 shows that, dependent on the choice of $\sigma_{\text {iw }}$ and $\Delta g^{\#}$, the estimated fit parameters differ. As an example, a contact angle estimate using CNT with, for example, $\sigma_{\text {iw }}$ from Pruppacher and Klett (2000) compared to using $\sigma_{\text {iw }}$ from Zobrist et al. (2007) leads to different results. In this section the sensitivity of two contact angle schemes to $\sigma_{\mathrm{iw}}$ and $\Delta g^{\#}$ is investigated.

The two CNT formulations used in this analysis are a single- $\alpha$ R\&D + fit $\Delta g^{\#}$ formulation (CNT2) and the $\alpha$ pdf R\&D + Z formulation (CNT5) described in Sect. 3.1. We chose these two formulations because CNT2 is used in GCMs and CNT5 to interpret our data. Both schemes contain two fit parameters ( $f$ and $\Delta g^{\#}$ in CNT2, $\mu$ and variance $\sigma$ of the contact angle distribution in CNT5).

We analyze how the two fit parameters depend on a change in thermodynamic and kinetic parameters. For this purpose the thermodynamic and kinetic parameters are varied up to $\pm 50 \%$. For each variation (e.g., an increase of $\sigma_{\text {iw }}$ by $10 \%$ ) fitting is done to the same immersion freezing data from Welti et al. (2012) as in the previous section. For the CNT2 the thermodynamic parameter $\sigma_{\mathrm{iw}}$ is varied; for the CNT5 the thermodynamic and kinetic parameters $\sigma_{\mathrm{iw}}$ and $\Delta g^{\#}$ are varied separately. The resulting fits are then compared to the reference fit results of Sect. 3.1 (see Table 1).

In both cases (CNT2 and CNT5) a similar change in fit parameters can be seen. Changing the thermodynamic parameter $\sigma_{\text {iw }}$ has a stronger impact on the fit parameters than changes in the kinetic parameter $\Delta g^{\#}$. This is expected from 


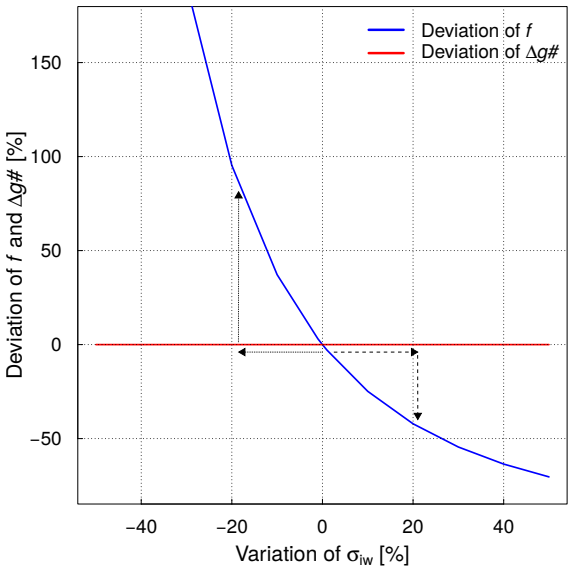

(a) Sensitivity analysis for the single- $\alpha \mathrm{R} \& \mathrm{D}+$ fit $\Delta g^{\#}$ formulation (CNT2)

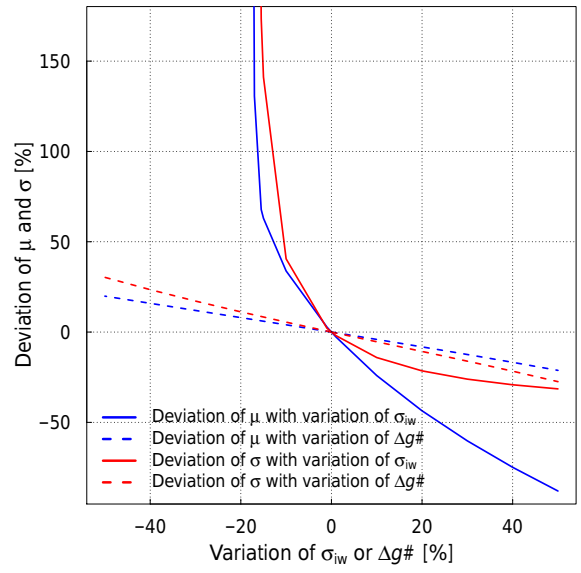

(b) Sensitivity analysis for the $\alpha$-pdf R\&D $+\mathrm{Z}$ formulation (CNT5)

Figure 4. Magnitude of deviation from a reference fit in percent (relative uncertainty) due to a variation of $\sigma_{\text {iw }}$ and $\Delta g^{\#}$ from 0 to $\pm 50 \%$. The applied change is indicated by the line type (solid is the change in thermodynamics, $\sigma_{\mathrm{iw}}$; dashed is the change in kinetics, $\Delta g^{\#}$ ).

the nucleation rate formula, where $\sigma_{\mathrm{iw}}$ enters the calculation of the nucleation rate to the power of three and therefore changes the nucleation rate or frozen fraction more drastically than a change in $\Delta g^{\#}$.

In case $\sigma_{\mathrm{iw}}$ is increased/overestimated, the fit parameters decrease to compensate the change (see dashed arrow in Fig. 4a) and conversely (see dotted arrow in Fig. 4a). The behavior of this compensation is not symmetric but follows the structure of the nucleation rate formula, i.e., $1 / x$ dependence for $f$ or $\mu$ and variance $\sigma$, respectively. That implies that the change in the fit parameter gets larger the larger the variation of $\sigma_{\mathrm{iw}}$ is. The relative change approaches negative $100 \%$ with increasing $\sigma_{\text {iw }}$. A larger deviation can be seen for the case where $\sigma_{\text {iw }}$ is decreased/underestimated.

Figure 4a shows the relative change of the fit parameters as a function of percental change in $\sigma_{\text {iw }}$ for CNT2. The higher the variation of $\sigma_{\text {iw }}$ is, the larger the deviation in $f$ from the reference fit value, whereas $\Delta g^{\#}$ remains unchanged. Only one fit parameter $(f)$ compensates for the change in $\sigma_{\text {iw }}$. Looking at Eqs. (1) and (4) the product of $f$ and $\sigma_{\mathrm{iw}}^{3}$ enters the exponential of the thermodynamic part of Eq. (4). As long as $f \times \sigma_{\text {iw }}^{3}$ is constant the resulting nucleation rate $J_{\text {imm }}$ is the same. Therefore $\Delta g^{\#}$ is not sensitive to a deviation of $\sigma_{\text {iw }}$.

Figure $4 \mathrm{~b}$ shows the relative change of the fit parameters as a function of percental change in $\sigma_{\text {iw }}$ and $\Delta g^{\#}$ for CNT5. In the case that $\Delta g^{\#}$ is changed (Fig. $4 \mathrm{~b}$ ) the compensation is linear for $\mu$, following the structure of the nucleation rate formula. For $\sigma$ the compensation is linear for small changes of $\Delta g^{\#}$ (until a change of approximately $30 \%$ ) but is nonlinear for larger variations of $\Delta g^{\#}$.

Summarizing, an over/underestimation of $\sigma_{\text {iw }}$ has a strong effect on the value of the resulting fit parameter, while the effect of over/underestimation of $\Delta g^{\#}$ is small. If fit parameters were estimated based on fitting different CNT formulations, they cannot be directly compared. Figure 4 can be used to estimate how different fit parameters would change due to different assumptions for $\sigma_{\text {iw }}$ or $\Delta g^{\#}$. An example of how fit parameters change with different CNT formulations is given in Appendix B.

\section{Strategy to evaluate different CNT formulations}

The sensitivity analysis in Sect. 3 shows that most CNT formulations with at least two fit parameters are able to reproduce measured freezing data. Contact angle schemes are mostly judged based on the RMSE of the estimated freezing curve and measured freezing data. Only looking at the reproducibility of freezing data, however, is not a conclusive measure of how well immersion freezing is represented, since the fit parameters can mask uncertainties in the thermodynamic and kinetic parameters of CNT. We propose to evaluate different CNT formulations by testing different fit properties against measurements. The evaluation consists of a macroscopic and a microscopic perspective. Three evaluation criteria are suggested.

At a macroscopic level, is the CNT formulation capable of reproducing the measured $T$-dependence of $F F$ and how well is dependence on IN size and time captured? According to the sensitivity of immersion freezing on $T, r_{\mathrm{IN}}$, and $t$, the representation of the temperature dependence, followed by the size of the IN and the predicted time dependence of the freezing process, should be captured by a suitable CNT formulation if it is used as a function of $T, r_{\mathrm{IN}}$, and $t$ in a GCM.

When evaluating fits to a dataset that contains freezing data as a function of $T, r_{\mathrm{IN}}$, and $t$ the goodness of the fit implicitly includes all three aspects. However, because $T$ has 
the strongest effect on the $F F$, the goodness of fit mostly reflects how well the CNT captures the temperature dependence. Two criteria emerge from the macroscopic level.

- Criterion 1: how accurately can the overall freezing data be reproduced, i.e., how well is the temperature dependence of the $F F$ captured by the CNT formulation?

- Criterion 2: how accurately are the particle size and time dependence of the freezing process captured by the CNT formulation? Criterion 2 can only be investigated when time- and particle-size-dependent measurements are available.

At a microscopic level, do the fit parameters match the microphysical assumptions of CNT and are they in general physically reasonable? To evaluate if fit parameters are physically reasonable, the analysis of heterogeneous freezing can be combined with findings from homogeneous freezing. Including homogeneous freezing into the analysis might be useful because of fewer unconstrained parameters in this case. The microscopic criterion to be tested is

- criterion 3: are the values for the fit parameters reasonable in the context of what we know about the microphysical process of nucleation?

In the following these three criteria are used to decide which CNT formulations are suitable for parameterizing immersion freezing, e.g., in a GCM.

\section{Using experimental data to estimate CNT parameters for different contact angle schemes}

In the following a comprehensive dataset of $F F$ (different aerosol species, aerosol particle sizes, and residence times in the experiment) is used. Five different mineral dust types were chosen for the analysis: Fluka kaolinite, illite-NX, montmorillonite, microcline (K-feldspar), and ATD (Arizona test dust). Montmorillonite or kaolinite are often used in global models as a surrogate for mineral dust in terms of ice nucleation, e.g., montmorillonite in ECHAM6-HAM2. Fluka kaolinite, which was used here, has been widely used to study the mechanism of immersion freezing. Illite-NX was chosen as a the mineral dust reference sample for an instrument intercomparison (Hiranuma et al., 2015). A microcline sample from Namibia and ATD are included to enable sensitivity studies of the freezing parameterization scheme with more efficient IN. The experimental data for kaolinite are taken from Welti et al. (2012). Illite-NX data have been published in Hiranuma et al. (2015). Microcline, montmorillonite, and ATD data are new datasets. All measurements were performed using size-selected aerosol particles with diameters of $50,100,200,400,800$, and $920 \mathrm{~nm}$ and $10 \mathrm{~s}$ residence time. The kaolinite dataset contains time-dependent measurements for different residence times of $1,2,3,6,9$, and
$21 \mathrm{~s}$. Note that the residence times are rounded to full seconds (compared to Welti et al., 2012) and not all datasets include the smallest and/or largest size (kaolinite: $100-920 \mathrm{~nm}$, illite $100-800 \mathrm{~nm}$, montmorillonite $100-800 \mathrm{~nm}$, microcline 50 $800 \mathrm{~nm}$, ATD 100-800 nm). The error bars of the data reflect the detection uncertainty and the statistical uncertainty in the measurement by multiple measurements.

To derive fit parameters for the CNT parameterization scheme, four CNT formulations are chosen: No. 1 (single$\alpha$ scheme), No. 2 (single- $\alpha$ scheme with $\Delta g^{\#}$ as a fit parameter), No. 5 ( $\alpha$-pdf scheme), and No. $7(\alpha(T)$ scheme). For more details see Table 1. CNT3, CNT4, and CNT6 use a $\sigma_{\text {iw }}$, which was found to not represent homogeneous freezing well, and are excluded based on criterion 3 . The wrong assumption of $\sigma_{\text {iw }}$ was chosen on purpose for the sensitivity study in Sect. 3.1 to demonstrate how that influences the fit results and the freezing curves. Note that also the single- $\alpha$ $\mathrm{R} \& \mathrm{D}+\mathrm{Z}$ formulation (CNT1) is not expected to be able to reproduce the experimental freezing data (criterion 1). It is still included here for comparison of the RMSE value with the other formulations ("bad" reference).

The fit parameters are determined by least-square minimization of the calculated versus measured $F F$. For this purpose the dataset of each dust species, including all measurements as a function of $T, r_{\mathrm{IN}}$, and $t$, is used. To get an impression of the variability of the fit parameters throughout a dataset, the kaolinite dataset is fitted for each size and time separately in Appendix C.

The fit parameters for the different CNT formulations and aerosol types are shown in Table 2 together with the best fit RMSE. The fit curves in comparison to the measured $F F$ are shown in Figs. 5 to 6 (in the case of kaolinite only a selection of the data is shown).

The geometric term $f$ in Table 2 is smallest for microcline, showing that this is the most efficient IN investigated here. The second lowest value for $f$ is found for ATD. Montmorillonite and kaolinite seem to be quite similar in terms of IN efficiency, whereas illite is the least efficient IN.

Revising the fit results with criterion 1 shows that CNT1 is too steep and not able to reproduce experimental data, resulting in a high RMSE. One fit parameter is not enough to shift and reduce the steepness of the immersion freezing curve sufficiently compared to homogeneous freezing. Thus the single- $\alpha$ scheme (CNT1) does not fulfill criterion 1. Reasonable fit results (low RMSE) are obtained with CNT2 and CNT5 for all datasets.

In the case of CNT5 the mean contact angle is very similar to the contact angle of CNT1, i.e., the medium freezing temperature $(F F=0.5)$ is similar, but the steepness of the freezing curve is reduced by the variance of the contact angle distribution $\sigma$. See Appendix A for additional analysis on the influence of $\mu$ and $\sigma$ on the fit of $F F$.

When fitting CNT7 more than one solution for fit parameters is found (no absolute minimum of the fitting function). However, $\alpha_{0}$ should be positive and $m$ has to be negative so 
Table 2. Derived fit parameters for the different CNT formulations and five different mineral dust types (kaolinite (kao), illite (ill), montmorillonite (mont), microcline (micro), ATD). The values are rounded to two digits after the decimal point. The logarithmic mean contact angle $\mu$ and the temperature-dependent contact angles are converted into the geometric term $f$ using Eq. (3).

\begin{tabular}{|c|c|c|c|c|c|c|c|c|c|}
\hline No. & Fit parameter & Kao & RMSE & Ill & RMSE & Mont & RMSE & Micro & RMSE \\
\hline 1 & $f$ & 0.56 & 0.2 & 0.61 & 0.17 & 0.56 & 0.18 & 0.3 & 0.22 \\
\hline \multirow[t]{2}{*}{2} & $f$ & 0.29 & 0.14 & 0.36 & 0.14 & 0.28 & 0.09 & 0.11 & 0.1 \\
\hline & $\Delta g^{\#} / 10^{-20} \mathrm{~J}$ & 9.95 & & 8.93 & & 10.03 & & 11.97 & \\
\hline \multirow[t]{3}{*}{5} & $\mu$ & 0.5 & 0.09 & 0.54 & 0.13 & 0.5 & 0.15 & 0.25 & 0.13 \\
\hline & $\hat{=} f_{\text {mean }}$ & $\approx 0.56$ & & $\approx 0.61$ & & $\approx 0.56$ & & $\approx 0.29$ & \\
\hline & $\sigma$ & 0.06 & & 0.05 & & 0.04 & & 0.11 & \\
\hline \multirow[t]{5}{*}{7} & $\alpha_{0} / \mathrm{rad}$ & 0.84 & 0.14 & 0.98 & 0.13 & 0.81 & 0.09 & 0.61 & 0.1 \\
\hline & $\hat{=} f_{260}$ & $\approx 0.18$ & & $\approx 0.27$ & & $\approx 0.17$ & & $\approx 0.14$ & \\
\hline & $\hat{=} f_{250}$ & $\approx 0.31$ & & $\approx 0.4$ & & $\approx 0.29$ & & $\approx 0.31$ & \\
\hline & $\hat{=} f_{240}$ & $\approx 0.45$ & & $\approx 0.55$ & & $\approx 0.43$ & & $\approx 0.53$ & \\
\hline & $m /\left(\operatorname{rad~K}{ }^{-1}\right)$ & -0.02 & & -0.02 & & -0.02 & & -0.03 & \\
\hline No. & Fit parameter & ATD & RMSE & & & & & & \\
\hline 1 & $f$ & 0.58 & 0.32 & & & & & & \\
\hline \multirow[t]{2}{*}{2} & $f$ & 0.14 & 0.21 & & & & & & \\
\hline & $\Delta g^{\#} / 10^{-20} \mathrm{~J}$ & 12.58 & & & & & & & \\
\hline \multirow[t]{3}{*}{5} & $\mu$ & 0.48 & 0.25 & & & & & & \\
\hline & $\hat{=} f_{\text {mean }}$ & $\approx 0.54$ & & & & & & & \\
\hline & $\sigma$ & 0.16 & & & & & & & \\
\hline \multirow[t]{5}{*}{7} & $\alpha_{0} / \mathrm{rad}$ & 0.39 & 0.13 & & & & & & \\
\hline & $\hat{=} f_{260}$ & $\approx 0.1$ & & & & & & & \\
\hline & $\hat{=} f_{250}$ & $\approx 0.31$ & & & & & & & \\
\hline & $\hat{=} f_{240}$ & $\approx 0.61$ & & & & & & & \\
\hline & $m$ & -0.04 & & & & & & & \\
\hline
\end{tabular}

Table 3. Ranking of the capability of the different CNT formulations to reproduce the freezing curves for different mineral dust particles based on the RMSE between the calculated freezing curve and the measured freezing data.

\begin{tabular}{lrrrrrr}
\hline No. & Kaolinite & Illite & Montmorillonite & Microcline & ATD & $\begin{array}{c}\text { Average } \\
\text { ranking }\end{array}$ \\
\hline 1 & 3 & 3 & 3 & 3 & 4 & 3.2 \\
2 & 2 & 2 & 1 & 1 & 2 & 1.6 \\
5 & 1 & 1 & 2 & 2 & 3 & 1.8 \\
7 & 2 & 1 & 1 & 1 & 1.2 \\
\hline No. & Average & Ranking based on & & & & \\
& RMSE & average RMSE & & & & \\
\hline 1 & 0.218 & 4 & & & \\
2 & 0.136 & 2 & & & & \\
5 & 0.15 & 1 & & & & \\
7 & 0.118 & & & & & \\
\hline
\end{tabular}

that $\alpha$ increases with decreasing $T$. Otherwise criterion 3 is not fulfilled. Here only the fit parameters that fulfill criterion 3 are reported (local minimum of the fitting function).

For all CNT formulations, the fits with largest RMSE are the ones for ATD which is probably caused by a sizedependent mineralogy of ATD. The CNT formulation that best reproduces immersion freezing varies from dust to dust. Therefore, we establish a ranking for each dataset similar to the methodology used in Wheeler et al. (2014). The best CNT formulation gets a ranking of 1, the worst a ranking of 4 . From the ranking of the different datasets an average ranking is derived to judge the overall capability to predict $F F$ for each CNT formulation. The ranking (see Table 3) shows that CNT7 and CNT2 are the best followed by CNT5 and CNT1. Calculating the average RMSE from all fits (as an alternative) leads to the same result, where CNT7 is the best and CNT1 


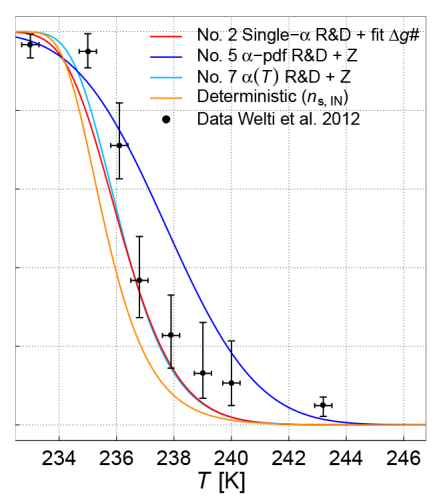

(a) $\mathrm{d}=100 \mathrm{~nm}, \mathrm{t}=10 \mathrm{~s}$

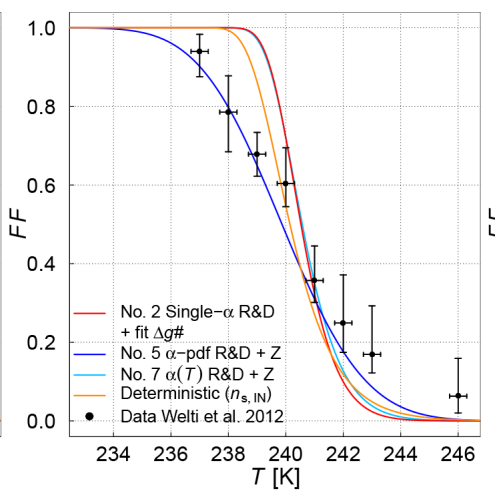

(b) $\mathrm{d}=800 \mathrm{~nm}, \mathrm{t}=10 \mathrm{~s}$

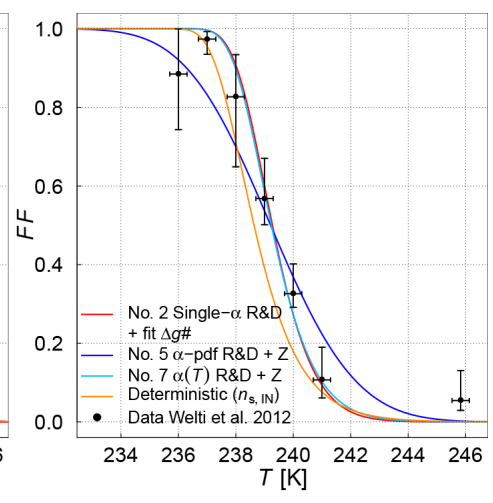

(c) $\mathrm{d}=400 \mathrm{~nm}, \mathrm{t}=10 \mathrm{~s}$

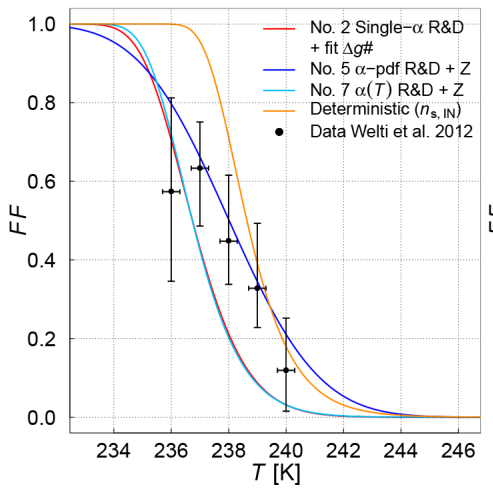

(d) d=400 nm, t=1 s

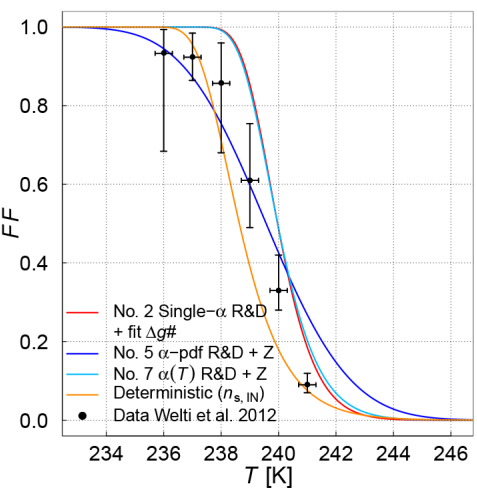

(e) $\mathrm{d}=400 \mathrm{~nm}, \mathrm{t}=21 \mathrm{~s}$

Figure 5. Calculated $F F$ of kaolinite for three different times and three sizes using the single- $\alpha$ R $\&$ D fit $\Delta g^{\#}$ formulation (CNT2), the $\alpha$-pdf R\&D $+\mathrm{Z}$ formulation (CNT5), and the $\alpha(T) \mathrm{R} \& \mathrm{D}+\mathrm{Z}$ formulation (CNT7) with corresponding fit parameters (see Table 2) and a simplified immersion freezing parameterization scheme based on Niemand et al. (2012) compared to the dataset. Panels (a-c) show the particle size dependence $(t=10 \mathrm{~s})$ and panels $(\mathbf{c}-\mathbf{e})$ show the time dependence $(d=400 \mathrm{~nm})$.

the worst (see also Table 3). Note that this ranking does not consider criteria 2 and 3 and is only based on fit statistics. It also does not show directly how good the CNT formulations reproduce time and particle size dependence of the freezing process (criterion 2).

In Sect. 5.1, the time and size dependence of the best three CNT formulations (CNT2, CNT5, and CNT7) are compared to the kaolinite dataset. A deterministic immersion freezing parameterization scheme based on Niemand et al. (2012) is included in the evaluation for comparison (for more details on the deterministic parameterization scheme see Appendix D). The Niemand et al. (2012) scheme is frequently used in literature for comparing laboratory measurements, e.g., Atkinson et al. (2013), Hoffmann et al. (2013), Kanji et al. (2013), O'Sullivan et al. (2014), Tobo et al. (2014), and Umo et al. (2015), but also as a parameterization scheme in model studies, e.g., Barahona et al. (2014), Paukert and Hoose (2014), and Hande et al. (2015).

\subsection{Testing the time and particle size dependence (criterion 2)}

To test the ability of the single- $\alpha$ R\&D + fit $\Delta g^{\#}$ formulation (CNT2), the $\alpha$-pdf R\&D $+\mathrm{Z}$ formulation (CNT5), and the $\alpha(T) \mathrm{R} \& \mathrm{D}+\mathrm{Z}$ formulation (CNT7) to reproduce experimentally observed size and time dependence, the fit parameters for kaolinite (see Table 2) are used to calculate $F F \mathrm{~s}$ for three different residence times $(1,10$, and $21 \mathrm{~s})$ and three different aerosol particle diameters $(100,400,800 \mathrm{~nm})$. In the case of the size-dependent calculation of $F F$ the time is $10 \mathrm{~s}$, while in the case of the time-dependent calculation the aerosol particle diameter is $400 \mathrm{~nm}$. The calculated $F F$ is compared to measurements of the size- and time-dependent $F F$ in Fig. 5. The analysis of the RMSE of the fit for each subset of data and CNT formulation revealed marginal differences in the second decimal place and is therefore not reported.

Figure 5 shows that CNT5 is able to capture the time and particle size dependence better than schemes 2 and 7 . This leads to an overall smaller RMSE and explains the better ranking for CNT5 in the case of kaolinite (see Table 2). Looking at Fig. 5, CNT2 and CNT7 give very similar results 


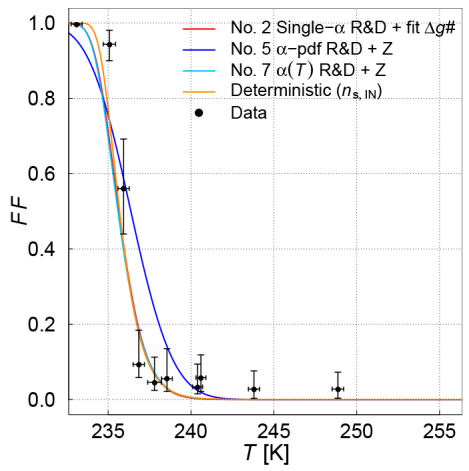

(a) Illite, $\mathrm{d}=100 \mathrm{~nm}$

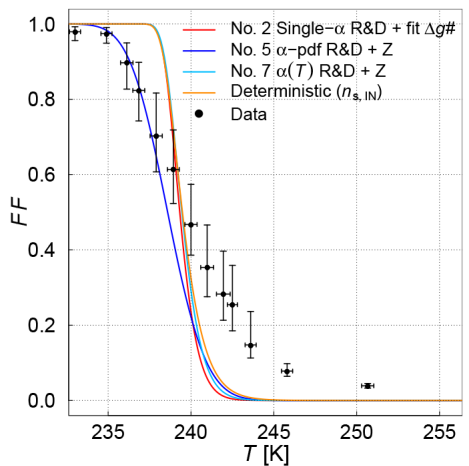

(d) Illite, $\mathrm{d}=800 \mathrm{~nm}$

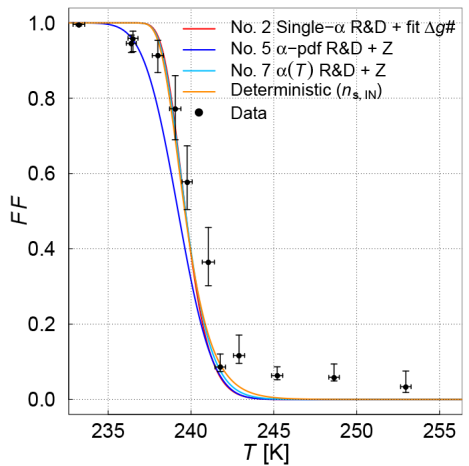

(g) Montmorillonite, $\mathrm{d}=400 \mathrm{~nm}$

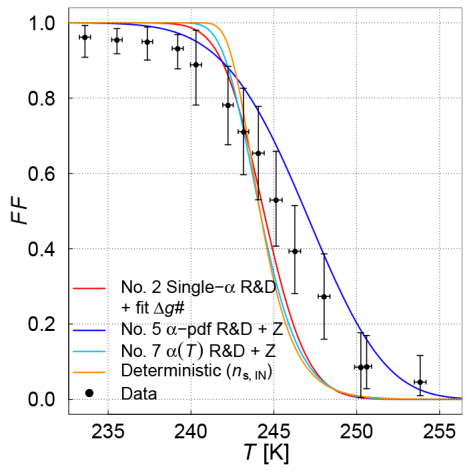

(j) Microcline, $d=100 \mathrm{~nm}$

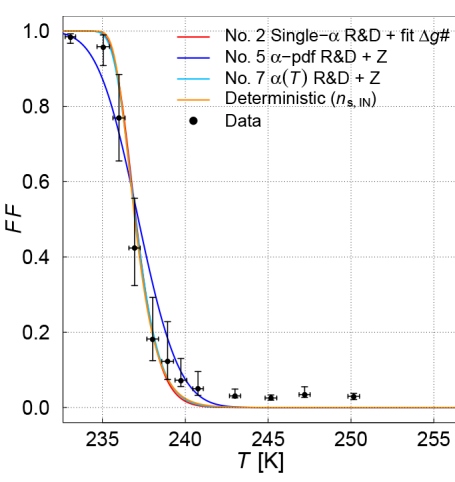

(b) Illite, $\mathrm{d}=200 \mathrm{~nm}$

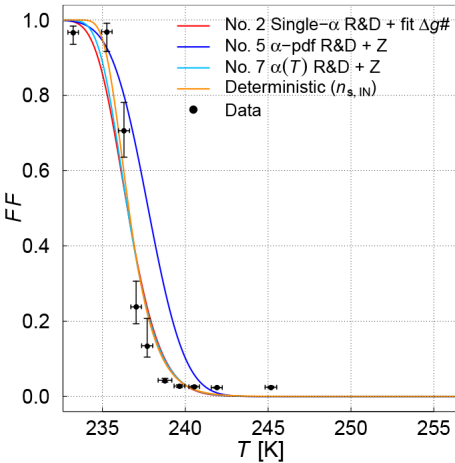

(e) Montmorillonite, $\mathrm{d}=100 \mathrm{~nm}$

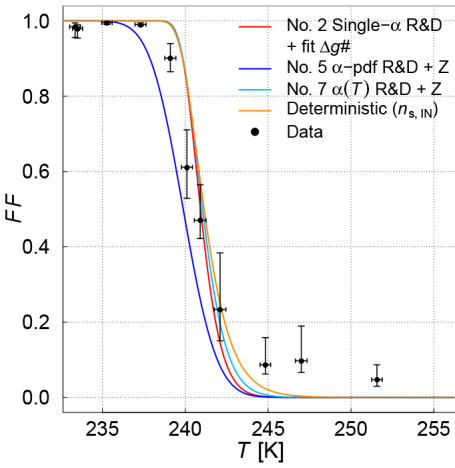

(h) Montmorillonite, $\mathrm{d}=800 \mathrm{~nm}$

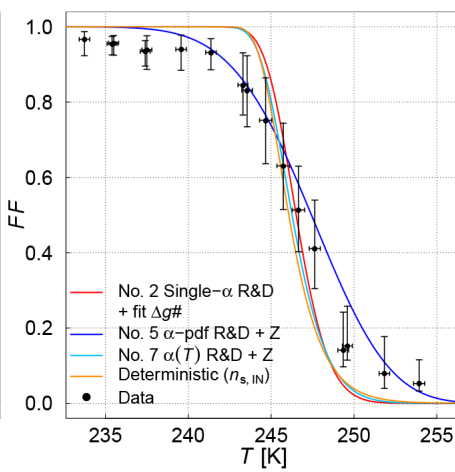

(k) Microcline, $\mathrm{d}=200 \mathrm{~nm}$

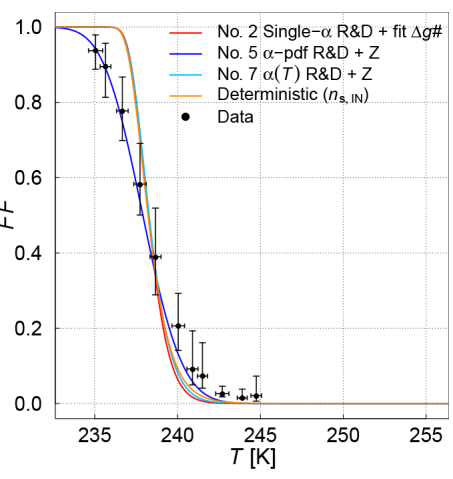

(c) Illite, $\mathrm{d}=400 \mathrm{~nm}$

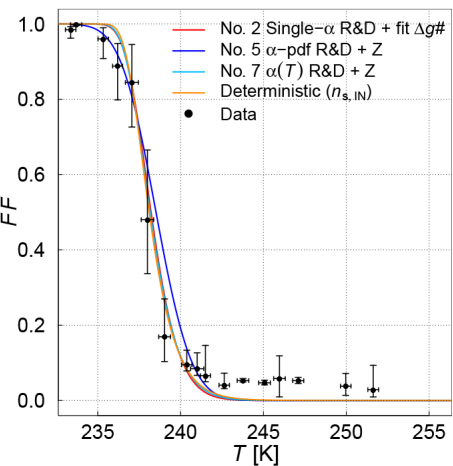

(f) Montmorillonite, $\mathrm{d}=200 \mathrm{~nm}$

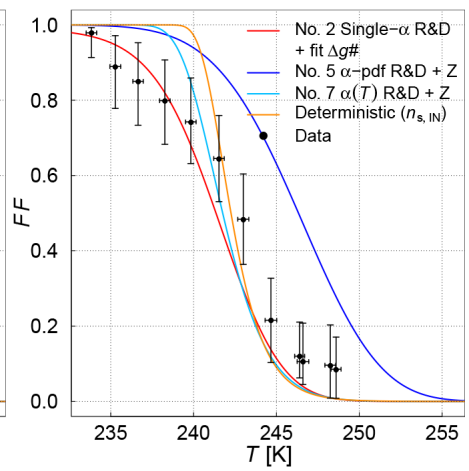

(i) Microcline, $\mathrm{d}=50 \mathrm{~nm}$

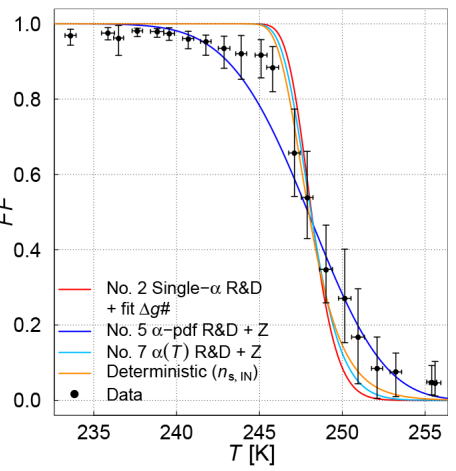

(l) Microcline, $d=400 \mathrm{~nm}$

Figure 6. 


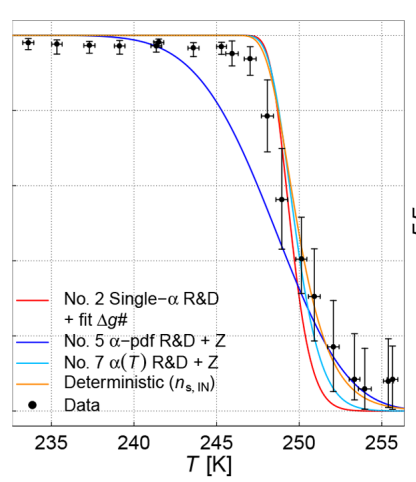

(m) Microcline, $\mathrm{d}=800 \mathrm{~nm}$

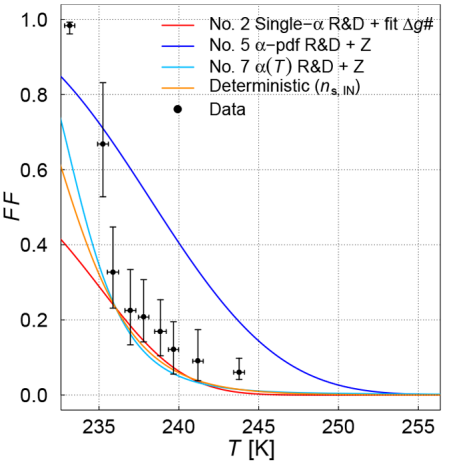

(n) ATD, d=100 nm

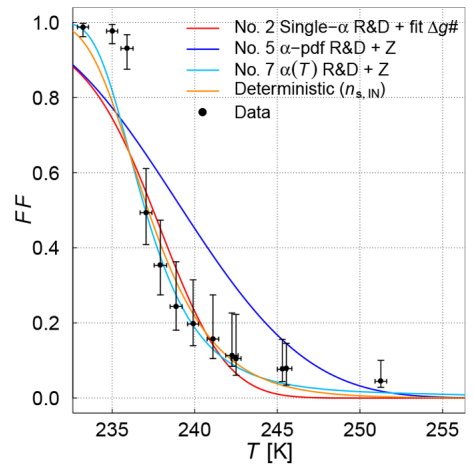

(o) ATD, $\mathrm{d}=200 \mathrm{~nm}$

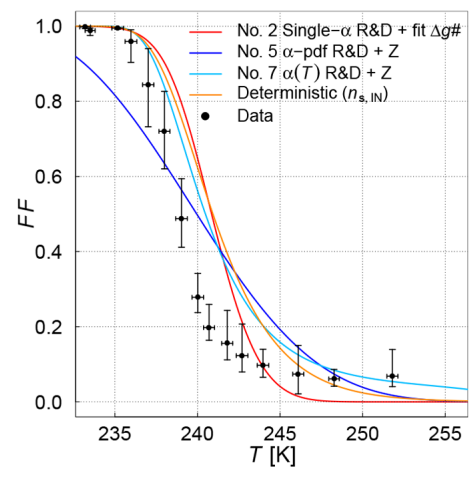

(p) ATD, d=400 nm

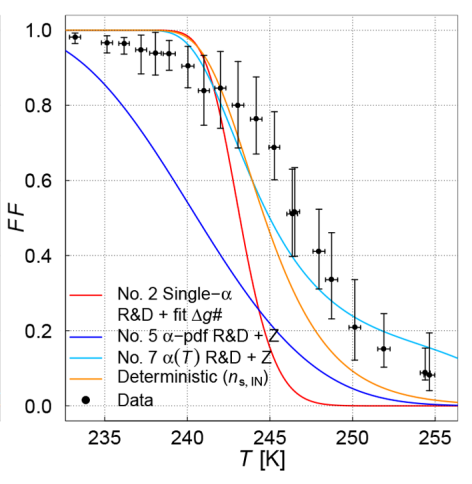

(q) ATD, d=800 nm

Figure 6. Calculated $F F$ of illite (a-d), montmorillonite (e-h), microcline (i-m), and ATD (n-q) after a residence time $t$ of $10 \mathrm{~s}$ for certain sizes using the single- $\alpha$ R\&D + fit $\Delta g^{\#}$ formulation (CNT2), the $\alpha$-pdf R\&D $+\mathrm{Z}$ formulation (CNT5), and the $\alpha(T)$ R\&D $+\mathrm{Z}$ formulation (CNT7) with corresponding fit parameters (see Table 2). A simplified immersion freezing parameterization scheme based on Niemand et al. (2012) is additionally compared to the dataset.

which overpredict both the size and time dependence, while CNT5 seems to underpredict the particle size dependence but captures the time dependence well. Overpredicting the size dependence translates into an overestimation of $F F$ for particles with an aerosol particle diameter larger than $400 \mathrm{~nm}$ and an underestimation of $F F$ for particles with an aerosol particle diameter smaller than $400 \mathrm{~nm}$. Underpredicting the size dependence has the opposite influence on $F F$. Overpredicting the time dependence means that $F F$ is overestimated when using a timestep larger than $10 \mathrm{~s}$ as in GCMs. The outcome of the evaluation depends on the dataset used. For different aerosol species the ranking of CNT5 and CNT2 differs, e.g., for montmorillonite, microcline, and ATD. Due to this limitation, it cannot generally be concluded which contact angle scheme best fulfills criterion 2. All three schemes might thus be chosen for CNT-based immersion freezing parameterization schemes in GCMs. The computationally least expensive formulations to use in a GCM would be CNT2 and CNT7.

In Fig. 5 the CNT curves are also compared to an empirical immersion freezing parameterization scheme ( $n_{\mathrm{s}, \mathrm{IN}}$ scheme) based on the expression given in Niemand et al. (2012) fitted to the kaolinite measurements (for details please see Ap- pendix D). The $n_{\mathrm{s}, \mathrm{IN}}$ scheme slightly overestimates the particle size dependence and the scheme does not include any time dependence since it is deterministic. Derived $F F$ curves appear similar to CNT curves. However, due to the general characteristics of empirical relations it is not clear whether it can be extrapolated to a wide $T$ range, which would be mandatory for the use in a GCM.

\subsection{Testing the physical reasonability of fit parameters (criterion 3)}

Since our knowledge about the microphysical behavior of supercooled water is limited, and measurements on the microscopic level are very difficult, the evaluation of the reasonability of the fit parameters is challenging. Homogeneous freezing measurements or results of molecular dynamics simulations can be used to additionally evaluate certain CNT formulations and contact angle schemes. Molecular simulations recently started to simulate heterogeneous freezing, often using kaolinite surfaces (Hu and Michaelides, 2007, 2008; Croteau et al., 2010; Šolc et al., 2011). Hu and Michaelides $(2007,2008)$ found that the ice nucleation ability of kaolinite results from the amphoteric nature of the surface hydroxyl $(\mathrm{OH})$ groups, meaning that these $\mathrm{OH}$ groups 
can act as hydrogen bond acceptors or donators. The surface $\mathrm{OH}$ groups are reacting flexibly to the orientation of the water molecules above the surface. Therefore water molecules bonding more easily with the kaolinite surface compared to other water molecules form an ice-like stable system. Such information can be used to understand the effect a particle surface can have on the kinetics of ice formation. Note that in molecular simulation studies sometimes the microscopic contact angle is estimated, which is the angle enclosed by the water surface and the substrate at their contact line. In case of a hydrophobic surface the microscopic contact angle is larger compared to the contact angle (equal when the contact angle is $90^{\circ}$ ). In case of a hydrophilic surface it is smaller.

The study of Šolc et al. (2011) is an example for a molecular simulation study that could help to evaluate fit parameters. Šolc et al. (2011) used force-field molecular dynamics simulation to investigate water nanodroplets on a kaolinite surface, which consists of tetrahedral silica sheets linked to octahedral alumina sheets. They estimated a contact angle of approximately $105^{\circ}$ on the tetrahedral sheets and approximately $0^{\circ}$ on the octahedral sheets (in the paper incorrectly named as microscopic contact angle). That is equivalent to a geometric term $f$ of 0.69 (Eq. 3) or 0. An average geometric term of a kaolinite surface containing these two types would be approximately 0.35 , which is similar to some of the experimentally derived values.

\subsection{Comparison to other studies}

Several previous studies investigated the capability of different CNT formulations to correctly reproduce measured freezing data for different IN, e.g., Zobrist et al. (2007), Marcolli et al. (2007), Lüönd et al. (2010), Niedermeier et al. (2011), Alpert et al. (2011), Knopf and Forrester (2011), Welti et al. (2012), Rigg et al. (2013), and Wheeler et al. (2014), some of them investigating contact angle schemes comparable to the ones in this study.

In all studies (including the present one) the single- $\alpha$ scheme does not represent the measured freezing data very well (only when $\Delta g^{\#}$ is used as an additional fit parameter). Even in the recent study of Alpert and Knopf (2016) the single- $\alpha$ scheme is not able to reproduce the freezing curve of a uniform surface (organic monolayer coating). The single- $\alpha+$ fit $\Delta g^{\#}$ scheme has not been evaluated against other schemes in the abovementioned studies. The ranking of the $\alpha(T)$ and $\alpha$-pdf schemes differs from study to study. In Zobrist et al. (2007), Alpert et al. (2011), Knopf and Forrester (2011), Welti et al. (2012), and Rigg et al. (2013) an $\alpha(T)$ scheme leads to good results. For all investigated IN (kaolinite, organic IN, and marine biogenic IN) $\alpha$ is increasing with decreasing $T$ in the mentioned literature. Note that in the study of Zobrist et al. (2007), Alpert et al. (2011), Knopf and Forrester (2011), and Rigg et al. (2013) the temperature dependence of $\alpha$ is based on the change in interfacial tensions with $T$ (according to Young, 1974).
The $\alpha$-pdf scheme did not produce good results in the study of Rigg et al. (2013) (aqueous ammonium sulfate droplets). They suggest that in case the $\alpha$-pdf scheme is used, it should be expressed as a function of water activity. In the study of Wheeler et al. (2014) it leads to good results for ATD, but less so for kaolinite. In both cases the best scheme was the active sites scheme, which was not investigated in the present study. In Welti et al. (2012) as well as in the present study the $\alpha$-pdf scheme reproduces the data quite well. Note that this study, the study of Lüönd et al. (2010) and Welti et al. (2012) are the only studies where the size and time dependence has been tested separately (criterion 2) as sizeand time-resolved data were available. From the results presented here, we conclude that the $\alpha$-pdf scheme is a suitable scheme to represent the time dependence for kaolinite. This could explain why it achieves a better ranking here than in Wheeler et al. (2014), where no size and time dependence could be tested against measurements. This paper includes the most complete evaluation (taking into account criteria 2 and 3 in addition to criterion 1) of contact angle schemes for immersion freezing of mineral dust types.

\section{Conclusions}

In this study the sensitivity of CNT-based immersion freezing parameterization schemes to thermodynamic and kinetic parameters is investigated. We discuss their effect on the fit parameters of contact angle schemes when fitting measurement data. For the use in models an assessment of sensitivities is important to estimate uncertainties originating from different parameterization schemes which represent the effect of aerosol particles on the energy barrier of ice nucleation.

Compared to homogeneous freezing, immersion freezing has one more unconstrained parameter, namely the geometric term $f(\alpha)$. Different schemes to represent the contact angle or contact angle distribution with one or two fit parameters based on experimental data are tested. It is found that contact angle schemes containing two fit parameters are able to reproduce experimental $F F$, while the contact angle scheme with only one fit parameter, the single- $\alpha$ scheme, cannot reproduce the freezing curves.

Analyzing the importance of the choice of $\sigma_{\text {iw }}$ and $\Delta g^{\#}$ to parameterize immersion freezing revealed that uncertainties in the thermodynamics or kinetics can be compensated by two-parameter contact angle schemes. As a result an under/overestimation of $\sigma_{\text {iw }}$ or $\Delta g^{\#}$ does not lead to a bad representation of freezing curves as in case of homogeneous freezing. Because the fit parameters compensate inaccuracies or uncertainties of the thermodynamic and kinetic parameters, the absolute value of the found fit parameters is highly dependent on the choice of thermodynamic and kinetic parameters within the formulation of CNT (especially on $\sigma_{\mathrm{iw}}$ ). As a consequence, contact angles for CNT parameterization schemes from different authors should only be applied within 
the same CNT formulation used to derive the parameters. Implementing a CNT-based parameterization scheme into a GCM demands that parameters derived from experiments are calculated using the same CNT formulation. Otherwise an offset of the freezing temperature in clouds might be introduced. The sensitivity of $\alpha$ on the thermodynamic and kinetic parameters used in CNT biases a direct comparison of contact angle values derived in different studies. We emphasize the importance of highlighting which CNT formulation was used for the analysis of experimental data.

Contact angle schemes with two or more fit parameters reproduce freezing curves but can be unphysical or limited to the dataset used for fitting. Contact angle schemes intended to represent immersion freezing properties of a heterogeneous particle population under a variety of environmental conditions and timescales should reproduce the $T$ dependence of freezing and the ability to predict the size and time dependence of the freezing process (criteria 1 and 2 ). The schemes should be conform to the microphysical assumptions CNT is based on (criterion 3). Particle size and nucleation time are implicitly included in the reproducibility of freezing curves of the dataset but should be investigated separately. It should be noted here that most experimental datasets are not accounting for both the size and time dependence of freezing. Experimental data without any information on the time or size dependence limit the assessment according to the criteria defined in this study. Here only a full analysis of the CNT parameterization schemes for one mineral dust (kaolinite) was possible. Having limited datasets may lead to unrepresentative conclusions. More size- and time-dependent measurements of different IN are desirable to compile parameters and find a robust $\mathrm{CNT}$ formulation.

In this study we derived fit parameters for five different datasets of mineral dust (kaolinite Fluka, illite-NX, montmorillonite, microcline, and ATD) by fitting different CNT formulations to the $F F$ from measurements. Good results in reproducing the freezing curves (criterion 1, $T$-dependence; partly criterion 2 , size and time dependence) are achieved when using a single- $\alpha$ scheme with fitted constant $\Delta g^{\#}$ (CNT2), an $\alpha$-pdf scheme (CNT5), or an $\alpha(T)$ scheme (CNT7).

\section{Data availability}

The underlying data for this publication can be found under the following doi:10.5905/ethz-1007-76 (Welti, 2015). 
Appendix A: Analysis of the different contact angle scheme formulations - how the fit parameters influence the calculated nucleation rate/frozen fraction

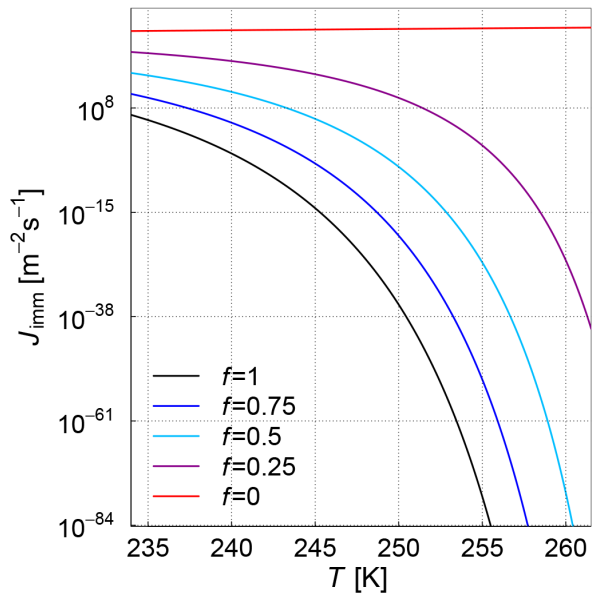

(a) CNT 1: $J_{\text {imm }}$ in dependence of $f$

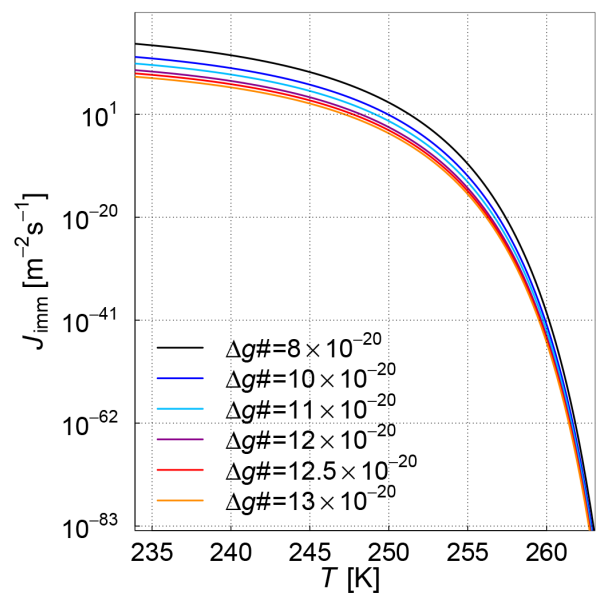

(c) CNT2: $J_{\text {imm }}$ in dependence of $\Delta g^{\#}$

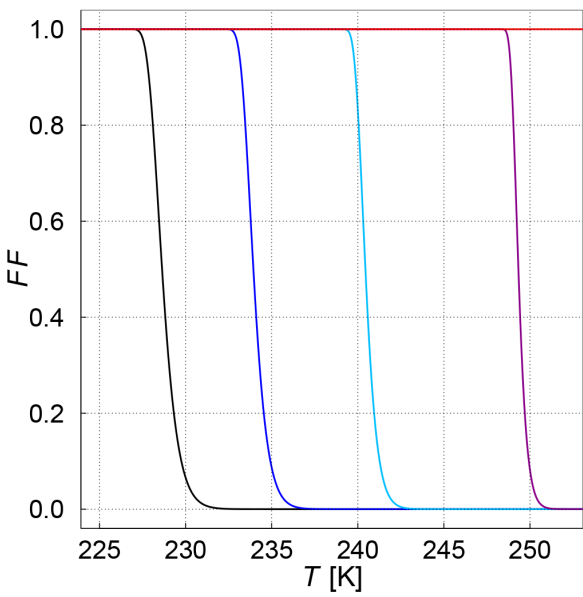

(b) CNT 1: $F F$ in dependence of $f$

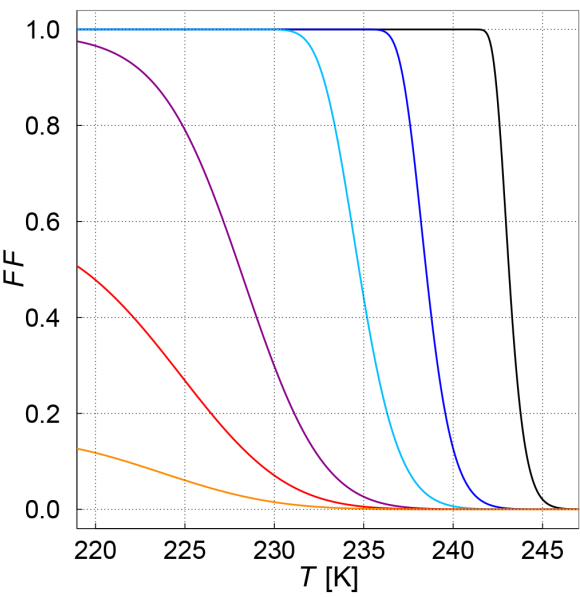

(d) CNT2: $F F$ in dependence of $\Delta^{\#} g$

Figure A1. Nucleation rate $J_{\mathrm{imm}}$ and frozen fraction $F F$ in dependence of the fit parameters for the contact angle schemes 1 and $2(f$ and $\Delta g^{\#}$ ). To estimate $F F$ we assume an aerosol particle diameter of $400 \mathrm{~nm}$ and a timestep of $10 \mathrm{~s}$. In case of the single- $\alpha$ R $\& \mathrm{D}+$ fit $\Delta g^{\#}$ scheme (No. 2) the geometric term $f$ was chosen to be 0.3 . The dependence of the formulation (in the case of a fixed $\Delta g^{\#}$ ) on $f$ is the same as with the single- $\alpha \mathrm{R} \& \mathrm{D}+\mathrm{Z}$ formulation (No. 1). Decreasing $f$ (reducing the energy barrier) shifts the freezing curve to higher temperatures while the slope slightly increases. Increasing $\Delta g^{\#}$ shifts the freezing curve to lower temperatures and changes the slope of the freezing curve. A higher activation energy barrier leads to a flattening of the curve. 


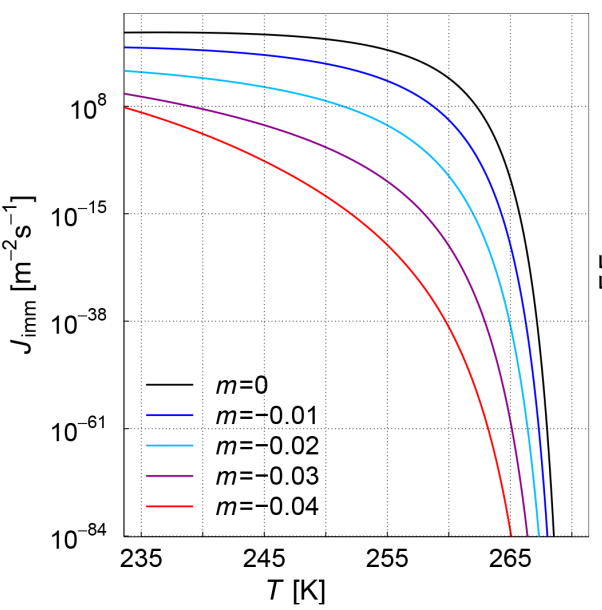

(a) CNT7: $J_{\text {imm }}$ in dependence of $m$

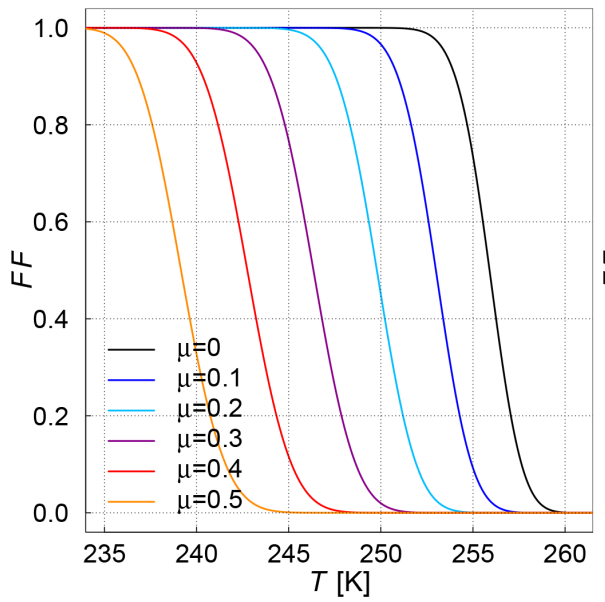

(c) CNT5: $F F$ in dependence of $\mu$

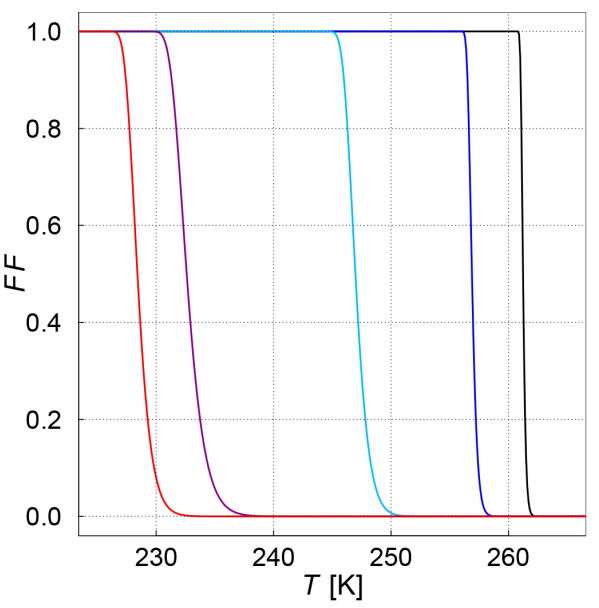

(b) CNT7: $F F$ in dependence of $m$

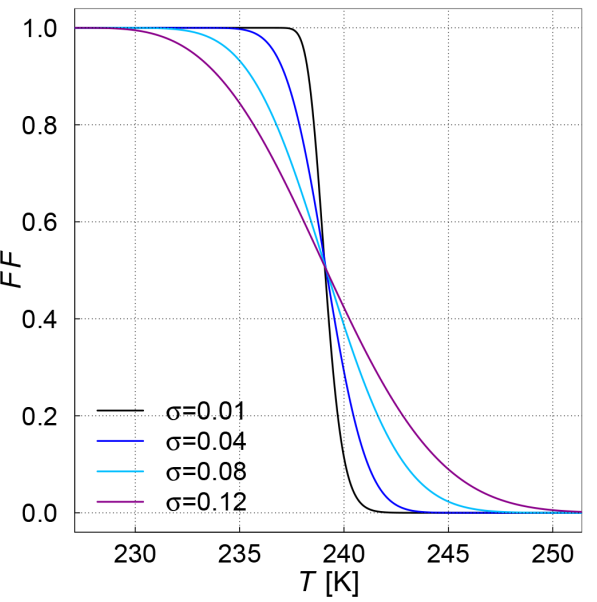

(d) CNT5: FF in dependence of $\sigma$

Figure A2. Nucleation rate $J_{\mathrm{imm}}$ and frozen fraction $F F$ in dependence of the fit parameters for the contact angle schemes 7 and $5(m, \mu$, and $\sigma$ ). To estimate $F F$ we assume a aerosol particle diameter of $400 \mathrm{~nm}$ and a timestep of $10 \mathrm{~s}$. In case of CNT7 $\alpha_{0}$ was chosen to be 0.8 $\left(\approx 46^{\circ}\right)$. Note that the dependence of the scheme on $\alpha_{0}$ (in the case of a fixed change in contact angle $m$ ) is the same as the change with $f$ of the single- $\alpha$ R\&D $+\mathrm{Z}$ formulation (CNT1). In case of the $\alpha$-pdf R\&D $+\mathrm{Z}$ formulation (CNT5) $\sigma$ was chosen to be 0.05 in the left figure and $\mu$ was chosen to be $0.5\left(\approx 29^{\circ}\right)$ in the right figure. Decreasing $m$ (increasing contact angle with decreasing $T$ because a broader part of the IN populations contact angle distribution causes nucleation) shifts the freezing curve to lower temperatures and changes the slope of the curve (flattening). Increasing $\mu$ (increasing the average contact angle) shifts the freezing curve to lower temperatures and slightly flattens the curve, while an increase in $\sigma$ (broadening of the contact angle distribution) changes the slope of the freezing curve only (flattening). Note that already small changes in $\mu$ lead to a considerable shift of the curve compared to the other schemes. 


\section{Appendix B: Estimating the relative uncertainty of fitting $\alpha$ - example calculations}

Figure 4 can be used to estimate the deviation of fit parameters from different CNT formulations relative to each other. To show this, we estimate the difference in fit parameter when using $\sigma_{\text {iw }}$ from Eadie (1971) instead of Reinhardt and Doye (2013) in combination with an $\alpha$-pdf scheme (CNT6 compared to CNT5).

Within the $10 \mathrm{~K}$ temperature range of the immersion freezing measurements (236-246 K) $\sigma_{\text {iw }}$, is on average $4 \%$ higher when using $\sigma_{\text {iw }}$ from Eadie (1971) instead of Reinhardt and Doye (2013). From Fig. $4 \mathrm{~b}$ we see that an increase in $\sigma_{\text {iw }}$ by $2.5 \%(246 \mathrm{~K})$ or $5 \%(236 \mathrm{~K})$ would lead to a decrease in $\mu$ by approximately 7 to $13 \%$. Now we check whether that estimated change matches with the real change when fitting the same dataset with the two different $\sigma_{\text {iw }}$. In Table 1 , using $\sigma_{\text {iw }}$ from Eadie (1971) leads to a mean contact angle of $0.44 \mathrm{rad}$ (approximately $25.5^{\circ}$ ) instead of $0.5 \mathrm{rad}$ (approximately $28^{\circ}$ ) when using $\sigma_{\text {iw }}$ from Reinhardt and Doye (2013). This is a difference of $12 \%$, conform with the estimate from Fig. 4 (approximately 7-13\%). However, the variance $\sigma$ of the $\alpha$ pdf distribution is expected to change less (5 to $9 \%$ ) but a change by $25 \%$ is found for the best fit.

In some cases the predicted change in fit parameters from Fig. 4 deviates from the real change in fit parameters (Table 1) because both parameter $\mu$ and $\sigma$ are changed at the same time. For Fig. 4 one was held constant. Another problem with Fig. 4 is that the assumption of a constant variation of $\sigma_{\text {iw }}$ over the whole temperature range is invalid in most cases. However, Fig. 4 can be used to illustrate how fit results might change and estimate a rough deviation from the reference when using different thermodynamic and kinetic parameters especially for cases where $\sigma_{\mathrm{iw}}$ changes nearly constant over the fitted temperature range. This can help when comparing fit results to fit results from studies where a different formulation of CNT was used.

\section{Appendix C: Variability of the fit parameters throughout one dataset}

The variability of the fit parameters throughout the dataset can be seen when fitting the single- $\alpha$ R\&D + fit $\Delta g^{\#}$ formulation (CNT2), the $\alpha$-pdf R\&D + Z formulation (CNT5), and the $\alpha(T) \mathrm{R} \& \mathrm{D}+\mathrm{Z}$ formulation (CNT7) to $F F$ of kaolinite data for different sizes and residence times separately. The resulting fit parameters are compared in Fig. $\mathrm{C} 1$ for different sizes in red and for different times in blue and light blue. Each point in Fig. $\mathrm{C} 1$ represents the value of the best fit parameter for one subset of the kaolinite dataset. The labels on the $x$ axis give information about which subset of the dataset was fitted. The residence time is $10 \mathrm{~s}$ for the data subsets of different sizes and the diameter of the kaolinite particles 400 or $800 \mathrm{~nm}$ for the data subsets of different times (blue/light blue). The dashed line indicates the mean of the size- or timedependent fit parameter. The range is shown as shaded box.

The fit parameters vary depending on the measurement conditions. Omitting the measurement with the shortest residence time $(1 \mathrm{~s})$ the variation between the data subsets for different times is small. The variability of the fit parameters is larger for different aerosol particle sizes compared to different residence times, which might be due to the higher sensitivity of the freezing process to particle size compared to time. For CNT2 the fit parameters seem to be correlated. High values of one fit parameter, e.g., $f$, correspond to low values of the other fit parameter, e.g., $\Delta g^{\#}$. CNT5, in contrast, does not show a clear correlation for the timedependent subsets but does for the size-dependent subsets. The same yields for CNT7.

The different fit results for CNT5 can be used to study how the shape of the contact angle distribution might change with the size of the particles or the residence time. Whereas the fitted contact angle distribution does not change noticeable with time between 1 and $21 \mathrm{~s}$ (figure not shown here), the mean contact angle $\left(e^{\mu}\right)$ and the variance $\sigma$ change with particle size (see Fig. C2). For all mineral dust types investigated here the contact angle distribution is shifted to the left (smaller contact angles) with increasing size, which means that the mean freezing efficiency of the IN population increases with size. Additionally for all mineral dust types except microcline the contact angle distribution broadens with increasing aerosol particle size (neglecting the fit of the $400 \mathrm{~nm}$ dataset of kaolinite, which does not fit into the picture). This reflects a larger probability of different $\alpha$ on the aerosol particle population with increasing size. The particle population is more heterogeneous. In the case of microcline the contact angle distribution narrows with increasing aerosol particle size, the ice nucleating properties of the microcline aerosol population seem to get more homogeneous with size. Note that the curves (here shown for kaolinite and microcline) are not considering measurement uncertainties of the fitted data and therefore can only be used to qualitatively interpret the result. In case of idealized measurements the result could be used to derive a relationship between mean contact angle $\left(e^{\mu}\right)$ or the width of the contact angle distribution $\sigma$ and the size of the IN. Using the results of Fig. $\mathrm{C} 1$ and developing a size-dependent $\alpha$-pdf improves the fit results.

\section{Uncertainty of fit parameters due to limited data}

In many cases no size- or time-dependent measurements are available. Here we investigate the quality of fit parameters when only limited amount of data are available. For that purpose we use the kaolinite dataset (as this is the most extensive dataset available within this study) and use only subsets of the dataset to estimate the fit parameters. The quality of the gained fit parameters is then estimated by using the complete dataset and how well the freezing curves can be represented is seen (RMSE). We look at four different cases: 

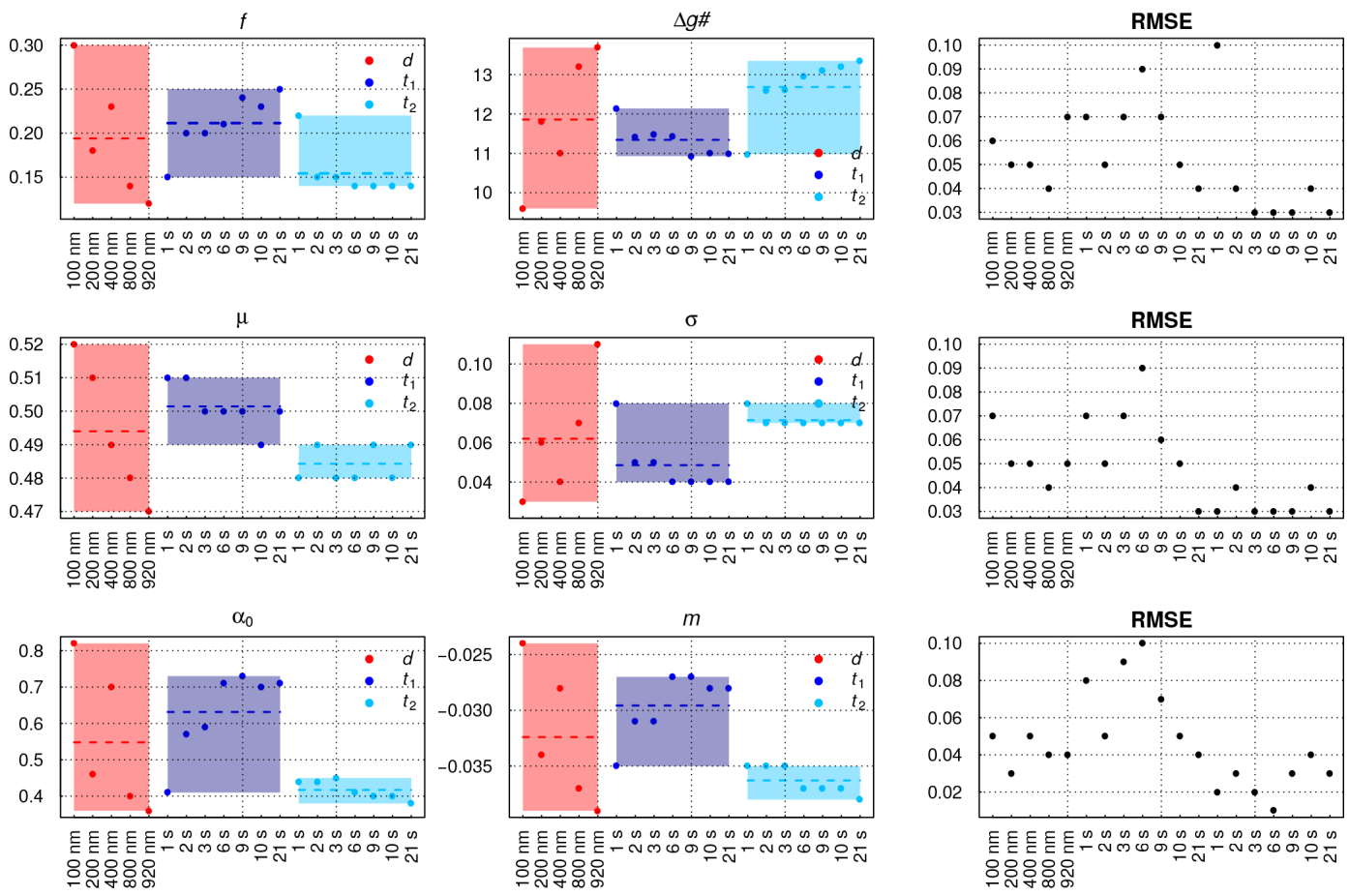

Figure C1. Variability of the fit parameters for subsets of the kaolinite dataset. The variability with the aerosol particle size $(d)$ is shown for a residence time $t$ of $10 \mathrm{~s}$ (red). The variability with time $\left(t_{1}\right.$ and $\left.t_{2}\right)$ is shown for a aerosol particle diameter of $400 \mathrm{~nm}\left(t_{1}\right.$, blue) and $800 \mathrm{~nm}$ ( $t_{2}$, light blue). The first row shows the fit results for CNT2, the second row for CNT5, and the third row for CNT7. The RMSE value shows the deviation of the fit to the single dataset it was fitted to.

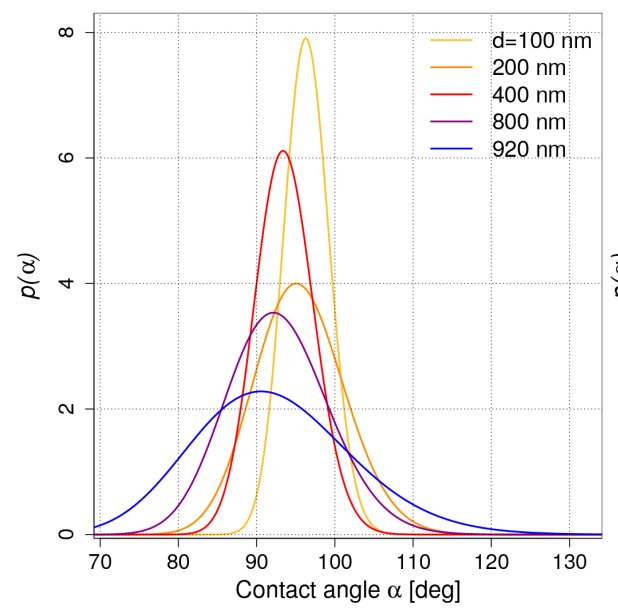

(a) Kaolinite

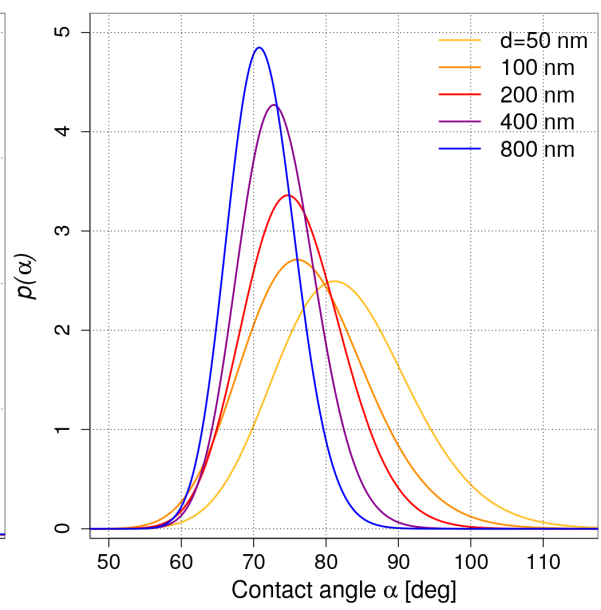

(b) Microcline

Figure C2. Change of $\alpha$-pdf with particle size for the kaolinite and microcline dataset. The residence time is $10 \mathrm{~s}$.

1. reference, in which the whole dataset is fitted (see also Table 2);

2. only size-dependent measurements are available, and time dependence is not known $(t=10 \mathrm{~s})$;

3. only time-dependent measurements are available, and size dependence is not known $(d=400 \mathrm{~nm})$;
4. only one measurement is available $(t=10 \mathrm{~s}, d=$ $400 \mathrm{~nm})$.

The resulting fit parameters and the deviation from the entire kaolinite dataset are shown in Table $\mathrm{C} 1$. The fit parameters are not significantly different when the dataset is limited to only size-dependent or only time-dependent data. Also, the deviation from the complete dataset is not significant. This 
Table C1. Derived fit parameters for two different CNT formulations based on the complete kaolinite dataset or subsets of the data. The values are rounded to two digits after the decimal point. The RMSE value is the deviation of the fit to the complete dataset.

\begin{tabular}{llrrrrrrrr}
\hline No. & Fit parameter & All data & RMSE & $\begin{array}{r}\text { Only } \\
\text { size }\end{array}$ & RMSE & $\begin{array}{r}\text { Only } \\
\text { time }\end{array}$ & RMSE & $\begin{array}{r}\text { Only one } \\
\text { dataset }\end{array}$ & RMSE \\
\hline 2 & $f$ & 0.29 & 0.14 & 0.29 & 0.14 & 0.3 & 0.14 & 0.23 & 0.15 \\
& $\Delta g^{\#} / 10^{-20} \mathrm{~J}$ & 9.95 & & 9.93 & & 9.7 & & 11.01 & \\
5 & $\mu$ & 0.5 & 0.09 & 0.5 & 0.1 & 0.5 & 0.09 & 0.49 & 0.12 \\
& $\sigma$ & 0.06 & & 0.05 & & 0.07 & & 0.04 & \\
\hline
\end{tabular}

analysis therefore does not allow any conclusion how many dependencies, e.g., size and time, have to be taken into account to successfully fit freezing data. However, if using only one single dataset, the results for the fit parameters are different and the deviation from the measurements is higher. Note that the deviation when fitting only a single dataset could be larger when a dataset is chosen that is not similar to the average values of the dataset as in this case. This means that there is no guarantee that fits can be extrapolated/used in a universal way across different conditions.

\section{Appendix D: Estimating the surface site density $n_{\mathrm{s}, \mathrm{IN}}$}

The Niemand et al. (2012) scheme for immersion freezing of natural dust is a deterministic scheme based on the approach of Connolly et al. (2009). It is derived from measurements carried out at the Aerosol Interaction and Dynamics in the Atmosphere (AIDA) cloud chamber at KIT, Karlsruhe (a detailed description of the AIDA cloud chamber and the measurements can be found in Niemand et al., 2012). In this approach it is assumed that ice nucleation occurs on localized sites, called active sites. It is described as a function of $T$ and particle surface area $A_{\mathrm{IN}}$ because the amount of active sites is supposed to scale with particle size.

In Connolly et al. (2009) the change of the number of ice active aerosol particles in the size bin $j, N_{\mathrm{i}, j}$, with respect to $T$, is

$\frac{\mathrm{d} N_{\mathrm{i}, j}}{\mathrm{~d} T}=\left(N_{\mathrm{i}, j}-N_{\mathrm{tot}, j}\right) \times A_{j} \times k(T)$,

where $N_{\text {tot }, j}$ denotes the total number of aerosol particles in the size bin $j$ and $A_{j}$ the dust particle surface area in the same size bin. The surface site density of ice active sites $n_{\mathrm{s}, \mathrm{IN}}$ as a function of $T$ can be determined by integrating the factor $k(T)$ over the whole temperature range:

$n_{\mathrm{S}, \mathrm{IN}}(T)=\int_{T}^{0} k(T) \mathrm{d} T$.

Using Eqs. (D1) and (D2) the frozen fraction $F F$ can be expressed as a function of $T$ :

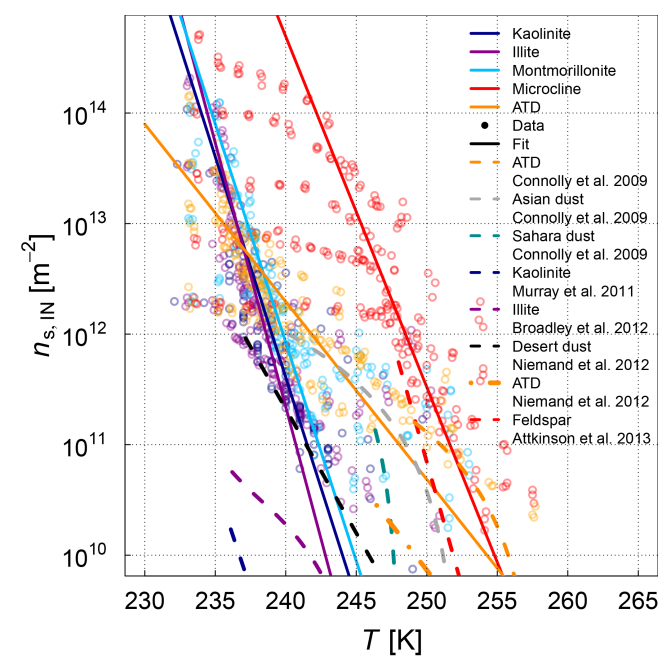

Figure D1. Surface site density $n_{\mathrm{s}, \mathrm{IN}}$ of different mineral dust types. The points are calculated surface site densities from $F F$ measurements, and the lines are the corresponding fits. The fits are based on FF (Eqs. D3 and D6 (method 1); Table D1, fifth row). Some estimates of $n_{\mathrm{s} \text {, IN }}$ found in literature are added. Colors indicate the dust type, and the line type indicates different literature studies.

$$
\begin{aligned}
F F & =\frac{N_{\mathrm{i}, j}}{N_{\mathrm{tot}, j}}=1-\exp \left(-A_{j} \times n_{\mathrm{s}, \mathrm{IN}}(T)\right) \\
& \approx A_{j} \times n_{\mathrm{s}, \mathrm{IN}}(T) .
\end{aligned}
$$

The approximation is valid for $A_{j} \times n_{\mathrm{s}, \mathrm{IN}}(T) \ll 1$, which translates into small particles and high temperatures. For low temperatures, such as, $243.15 \mathrm{~K}$ and particles larger than $3 \mu \mathrm{m}$ the term $A_{j} \times n_{\mathrm{s}, \mathrm{IN}}$ is approximately 1 .

The surface site density of ice active sites, $n_{\mathrm{s}, \mathrm{IN}}(T)$, is calculated from the total surface area of aerosol particles in the AIDA chamber and the measured ice crystal number concentration during one freezing experiment:

$$
\begin{aligned}
& \sum_{j=1}^{n} N_{\mathrm{i}, j} \approx \sum_{j=1}^{n} N_{\mathrm{tot}, j} \times A_{j} \times n_{\mathrm{s}, \mathrm{IN}}(T)=n_{\mathrm{s}, \mathrm{IN}}(T) \\
& \times \sum_{j=1}^{n} N_{\mathrm{tot}, j} \times A_{j}
\end{aligned}
$$




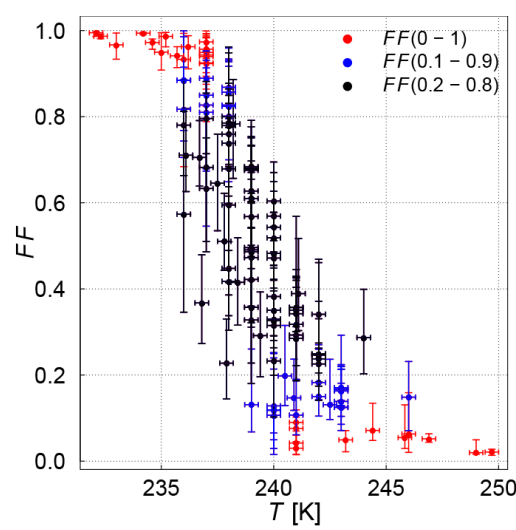

(a) Kaolinite $F F$ dataset.

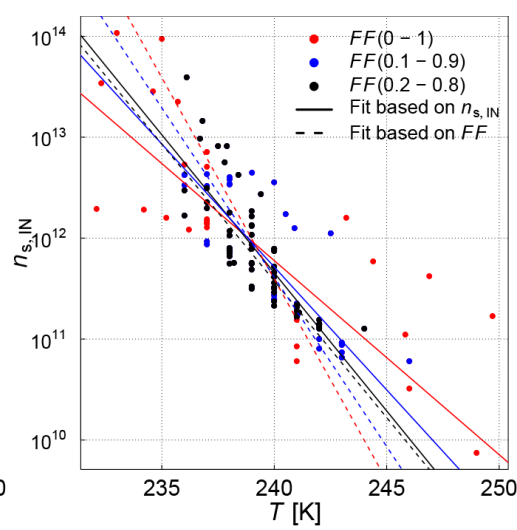

(b) Calculated $n_{\mathrm{s} \text {, IN }}$ based on the $F F$ dataset (points) and fits (lines).

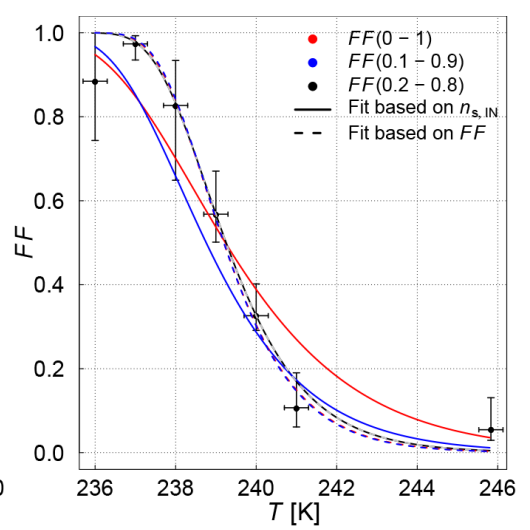

(c) One kaolinite dataset $(\mathrm{d}=400 \mathrm{~nm}, \mathrm{t}=10 \mathrm{~s})$ with calculated freezing curves using different $n_{\mathrm{S}, \mathrm{IN}}$.

Figure D2. Sensitivity study of different methods to estimate the surface site density $n_{\mathrm{s} \text {,IN }}$ of kaolinite and the resulting freezing curve. The data subsets are color coded as indicated in the legend.

$$
\begin{gathered}
\Leftrightarrow n_{\mathrm{s}, \mathrm{IN}}(T)=\frac{\sum_{j=1}^{n} N_{\mathrm{i}, j}}{\sum_{j=1}^{n} N_{\mathrm{tot}, j} \times A_{j}}=\frac{\sum_{j=1}^{n} N_{\mathrm{i}, j}}{\sum_{j=1}^{n} A_{\mathrm{tot}, j}} \\
=\frac{N_{\mathrm{i}}}{A_{\mathrm{tot}}}
\end{gathered}
$$

with $A_{\text {tot }, j}$ the total surface area per unit volume of particles in the size bin $j$ and $A_{\text {tot }}$ the total surface area over all size bins. The evaluation of 16 AIDA freezing experiments yields the following fit formula for the ice active surface site density of natural dust:

$$
\begin{aligned}
n_{\mathrm{s}, \mathrm{IN}}(T)\left(\mathrm{m}^{-2}\right) & =b \times \exp (-A \times a \\
& \times(T-273.15 K)+B),
\end{aligned}
$$

with the fit parameters $A=0.517$ and $B=8.934$ and the unit correction factors $a=\mathrm{K}^{-1}$ and $b=\mathrm{m}^{-2}$. Due to the temperature range of the freezing experiments, the parameterization is limited to the temperatures from 261.15 to $237.15 \mathrm{~K}$.

Equation (D6) was used here to fit the dataset for the different mineral dust types. The fits were done in two different ways: by using Eqs. (D3) and (D6) to fit the measured $F F$ (method 1) or by using Eq. (D3) to convert the $F F$ measurements to logarithmic surface site densities and fit $\ln \left(n_{\mathrm{s}, \mathrm{IN}}(T)\right)$ directly following Eq. (D6) (method 2). The results are shown in Table D1 and in Fig. D1 (method 1). The scheme is labeled " $n_{\mathrm{s}, \mathrm{IN}}$ " in Fig. 5.

Table D1 shows that the results for the fit parameters are very different depending on whether the $F F$ is fitted directly or the active site density $n_{\mathrm{S}, \mathrm{IN}}$ is calculated from the $F F$ and fitted afterwards. That is due to the characteristics of the freezing curve. The very small $F F$ at high temperatures and limited $F F$ (to 1) at low temperatures lead to a flattening of the $n_{\mathrm{s}, \mathrm{IN}}$ curve. Calculating $n_{\mathrm{s}, \mathrm{IN}}$ at low temperatures from $F F$ close to 1 gives the number of active sites, which was needed to freeze all droplets. However, it could be that more actives sites were present than needed to freeze all droplets. Therefore the tail (low and high $F F$ ) of the $F F$ dataset is often left out of the fitting. Here we investigate how the fit results and the freezing curves change depending on the share of the dataset accounted for fitting. We use the complete dataset as a first step and then omit $F F$ data higher than 0.9 and lower than 0.1 and 0.8 and 0.2 , respectively. Figure D2 shows this exemplary using a dataset of kaolinite particles $(d=400 \mathrm{~nm}, t=10 \mathrm{~s})$. In Fig. D2b it can be seen that the surface site density $n_{\mathrm{s}, \mathrm{IN}}$ varies depending on the share of the $F F$ dataset. The implication of the different estimations for $n_{\mathrm{s}, \mathrm{IN}}$ is shown in Fig. D2c.

The freezing curves from the indirect $n_{\mathrm{s}, \mathrm{IN}}$ fit (method 1) are not so different from each other and capture the measurements quite well. The variation of the estimated $F F$ due to the share of the $F F$ dataset, which was used for fitting, is larger when $n_{\mathrm{S}, \mathrm{IN}}$ is estimated directly by fitting calculated $n_{\mathrm{s}, \text { IN }}$ from $F F$ measurements (method 2). The largest deviation from all other fits originates when $n_{\mathrm{s}, \mathrm{IN}}$ is estimated directly taking all data into account. Cutting away the tail of the $F F$ measurements leads to a very similar result when $n_{\mathrm{s}, \mathrm{IN}}$ is estimated directly (black solid line) compared to the indirect estimate of $n_{\mathrm{s}, \mathrm{IN}}$ using the complete $F F$ data (red dashed line) (see also Table D1). When cutting away the tail of the $F F$ data $(F F>0.2$ and $F F<0.8)$, the freezing curve based on the direct estimated $n_{\mathrm{s}, \mathrm{IN}}$ also captures the data well and falls on the freezing curve of the indirect $n_{\mathrm{s}, \mathrm{IN}}$ using the same share of $F F$ data. We recommend cutting the tails from the $F F$ data when $n_{\text {s,IN }}$ is fitted directly. When estimating $n_{\mathrm{S}, \mathrm{IN}}$ indirectly by using $F F$ there is no need to cut the tail. Not cutting the tail increases the amount of data available for the fitting and might therefore be preferable. However, very low/high $F F$ are most susceptible to experimental uncertainties, which could be a legitimation for cutting the tail away 


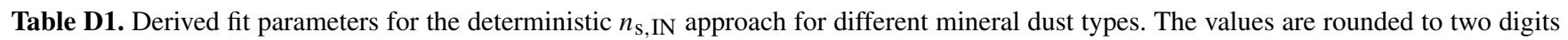
after the decimal point.

\begin{tabular}{|c|c|c|c|c|c|c|}
\hline Approach & $\begin{array}{l}\text { Fit } \\
\text { parameter }\end{array}$ & Kao & Ill & Mont & Micro & ATD \\
\hline$n_{\mathrm{s}, \mathrm{IN}}$ direct & $A$ & 0.44 & 0.37 & 0.34 & 0.29 & 0.28 \\
\hline (complete dataset) & $B$ & 12.44 & 15.18 & 16.98 & 20.78 & 19.14 \\
\hline$n_{\mathrm{S}, \mathrm{IN}}$ direct & $A$ & 0.56 & 0.7 & 0.7 & 0.52 & 0.3 \\
\hline (only $F F$ between 0.1 and 0.9 ) & $B$ & 8.38 & 3.69 & 4.61 & 15.16 & 18.43 \\
\hline$n_{\mathrm{s}, \mathrm{IN}}$ direct & $A$ & 0.63 & 0.76 & 0.81 & 0.65 & 0.29 \\
\hline (only $F F$ between 0.2 and 0.8 ) & $B$ & 5.95 & 1.29 & 0.88 & 11.73 & 18.63 \\
\hline$n_{\mathrm{s}, \mathrm{IN}}$ based on $F F$ & $A$ & 0.92 & 1.1 & 0.91 & 0.73 & 0.37 \\
\hline (complete dataset) & $B$ & -3.77 & -10.35 & -2.71 & 9.63 & 16.04 \\
\hline$n_{\mathrm{S}, \mathrm{IN}}$ based on $F F$ & $A$ & 0.77 & 1.04 & 0.9 & 0.73 & 0.27 \\
\hline (only $F F$ between 0.1 and 0.9 ) & $B$ & 1.13 & -8.3 & -2.11 & 9.49 & 19.19 \\
\hline$n_{\mathrm{s}, \mathrm{IN}}$ based on $F F$ & $A$ & 0.62 & 0.93 & 0.88 & 0.74 & 0.25 \\
\hline (only $F F$ between 0.2 and 0.8 ) & $B$ & 5.97 & -4.54 & -1.5 & 9.29 & 19.84 \\
\hline
\end{tabular}

from the dataset. Because the results are different depending on the methodology this sensitivity should be taken into account when comparing different fit parameters of $n_{\mathrm{s}, \mathrm{IN}}$ from literature. We recommend to estimate $n_{\mathrm{s}, \mathrm{IN}}$ indirectly by fitting $F F$ as a standard procedure. 


\section{Appendix E: List of symbols}

\begin{tabular}{|c|c|c|}
\hline Symbol & Unit & Description \\
\hline$A_{\text {IN }}$ & $\mathrm{m}^{2}$ & Surface area of an IN \\
\hline$A_{\text {tot }}$ & $\mathrm{m}^{-2}$ & Total surface area per unit volume of particles over all size bins \\
\hline$A_{\text {tot }, j}$ & $\mathrm{~m}^{-2}$ & Total surface area per unit volume of particles in size bin $j$ \\
\hline$A_{j}$ & $\mathrm{~m}^{-2}$ & Dust particle surface area in size bin $j$ \\
\hline$C_{\text {prefac }}$ & $\mathrm{m}^{-3} \mathrm{~s}^{-1}$ & Pre-exponential factor of the nucleation rate \\
\hline$d$ & $\mathrm{~m}$ & Aerosol particle diameter \\
\hline$e_{\mathrm{sw}}$ & $\mathrm{Pa}$ & Saturation vapor pressure over water; $e_{\mathrm{sw}}=611.2 \times \exp \left(\frac{17.62 \times(T-273.15 \mathrm{~K})}{T-30.3 \mathrm{~K}}\right)$ \\
\hline$e_{\mathrm{si}}$ & $\mathrm{Pa}$ & Saturation vapor pressure over ice; $e_{\mathrm{si}}=611.2 \times \exp \left(\frac{22.46 \times(T-273.15 \mathrm{~K})}{T-0.53 \mathrm{~K}}\right)$ \\
\hline$f$ & - & Geometric term \\
\hline$f_{\text {mean }}$ & - & Mean geometric term of the contact angle distribution \\
\hline$f_{260}, f_{250}, f_{240}$ & - & Geometric term at $T=260,250$ or $240 \mathrm{~K}$ \\
\hline$F F$ & - & Frozen fraction \\
\hline$h$ & $\mathrm{~J} \mathrm{~s}$ & Planck constant; $6.63 \times 10^{-34} \mathrm{~J} \mathrm{~s}$ \\
\hline$J_{\text {hom }}$ & $\mathrm{m}^{-3} \mathrm{~s}^{-1}$ & Homogeneous nucleation rate \\
\hline$J_{\mathrm{imm}}$ & $\mathrm{m}^{-2} \mathrm{~s}^{-1}$ & Immersion freezing nucleation rate \\
\hline$k_{\mathrm{B}}$ & $\mathrm{J} \mathrm{K}^{-1}$ & Boltzmann constant; $1.38 \times 10^{-23} \mathrm{~J} \mathrm{~K}^{-1}$ \\
\hline$n_{k, \text { germ }}$ & - & Number of water molecules in the ice germ \\
\hline$n_{\mathrm{S}}$ & $\mathrm{m}^{-2}$ & Number of water molecules in contact with the unit area of the ice germ; $5.85 \times 10^{16} \mathrm{~m}^{-2}$ \\
\hline$n_{\mathrm{s}, \mathrm{IN}}$ & $\mathrm{m}^{-2}$ & Surface density of active sites on an IN \\
\hline$N_{\mathrm{i}}$ & - & Ice crystal number concentration \\
\hline$N_{\mathrm{i}, j}$ & - & Number of ice active aerosol particles in size bin $j$ \\
\hline$N_{1}$ & $\mathrm{~m}^{-3}$ & Volume number density of a water molecule in liquid water \\
\hline$N_{\text {tot }, j}$ & - & Total number of aerosol particles in the size bin $j$ \\
\hline$m$ & $\operatorname{rad~K}{ }^{-1}$ & Change of contact angle with temperature \\
\hline$p(\alpha)$ & - & Probability density of contact angle $\alpha$ \\
\hline$r_{\text {germ }}$ & $\mathrm{m}$ & Radius of the ice germ ( $=$ critical radius) \\
\hline$r_{\mathrm{IN}}$ & $\mathrm{m}$ & Radius of the IN \\
\hline$S_{\mathrm{i}}$ & - & Saturation ratio with respect to ice; $S_{\mathrm{i}}=\frac{e_{\mathrm{sw}}}{e_{\mathrm{si}}}$ \\
\hline$t$ & $\mathrm{~s}$ & Time \\
\hline$T$ & $\mathrm{~K}$ & Temperature \\
\hline$v_{\text {ice }}$ & $\mathrm{m}^{3}$ & Volume of a water molecule in the ice embryo \\
\hline$Z$ & - & Zel'dovich factor \\
\hline$\alpha$ & $\mathrm{rad}$ & Contact angle \\
\hline$\alpha_{0}$ & $\mathrm{rad}$ & Contact angle at melting point \\
\hline$\Delta g^{\#}$ & $\mathrm{~J}$ & Activation energy barrier \\
\hline$\Delta G, \Delta G_{\mathrm{hom}}$ & $\mathbf{J}$ & Gibbs free energy barrier (of homogeneous freezing) \\
\hline$\Delta t$ & $\mathrm{~s}$ & Timestep \\
\hline$\mu$ & - & Natural logarithm of the mean contact angle of the contact angle distribution \\
\hline$\sigma$ & - & Variance of the contact angle distribution \\
\hline$\sigma_{\mathrm{iw}}$ & $\mathrm{J} \mathrm{m}^{-2}$ & Interfacial tension between ice/water \\
\hline$\pi$ & - & Ratio of a circle's circumference to its diameter \\
\hline
\end{tabular}


Acknowledgements. The authors would like to thank the anonymous reviewers, P. A. Alpert, C. Hoose, and P. Connolly for interesting discussions and their valuable input. Furthermore, we thank S. Ferrachat for the help with estimating the computational costs for extra tracers.

Edited by: A. Nenes

Reviewed by: two anonymous referees

\section{References}

Alpert, P. A. and Knopf, D. A.: Analysis of isothermal and coolingrate-dependent immersion freezing by a unifying stochastic ice nucleation model, Atmos. Chem. Phys., 16, 2083-2107, doi:10.5194/acp-16-2083-2016, 2016.

Alpert, P. A., Aller, J. Y., and Knopf, D. A.: Initiation of the ice phase by marine biogenic surfaces in supersaturated gas and supercooled aqueous phases, Phys. Chem. Chem. Phys., 13, 19882-19894, doi:10.1039/C1CP21844A, 2011.

Ansmann, A., Tesche, M., Seifert, P., Althausen, D., Engelmann, R., Fruntke, J., Wandinger, U., Mattis, I., and Müller, D.: Evolution of the ice phase in tropical altocumulus: SAMUM lidar observations over Cape Verde, J. Geophys. Res.-Atmos., 114, D17208, doi:10.1029/2008JD011659, 2009.

Atkinson, J. D., Murray, B. J., Woodhouse, M. T., Whale, T. F., Baustian, K. J., Carslaw, K. S., Dobbie, S., O'Sullivan, D., and Malkin, T. L.: The importance of feldspar for ice nucleation by mineral dust in mixed-phase clouds, Nature, 498, 355-358, 2013.

Barahona, D.: Thermodynamic derivation of the activation energy for ice nucleation, Atmos. Chem. Phys., 15, 13819-13831, doi:10.5194/acp-15-13819-2015, 2015.

Barahona, D., Molod, A., Bacmeister, J., Nenes, A., Gettelman, A., Morrison, H., Phillips, V., and Eichmann, A.: Development of two-moment cloud microphysics for liquid and ice within the NASA Goddard Earth Observing System Model (GEOS-5), Geosci. Model Dev., 7, 1733-1766, doi:10.5194/gmd-7-17332014, 2014.

Broadley, S. L., Murray, B. J., Herbert, R. J., Atkinson, J. D., Dobbie, S., Malkin, T. L., Condliffe, E., and Neve, L.: Immersion mode heterogeneous ice nucleation by an illite rich powder representative of atmospheric mineral dust, Atmos. Chem. Phys., 12, 287-307, doi:10.5194/acp-12-287-2012, 2012.

Cabriolu, R. and Li, T.: Ice nucleation on carbon surface supports the classical theory for heterogeneous nucleation, Phys. Rev. E, 91, 052402, doi:10.1103/PhysRevE.91.052402, 2015.

Chen, J.-P., Hazra, A., and Levin, Z.: Parameterizing ice nucleation rates using contact angle and activation energy derived from laboratory data, Atmos. Chem. Phys., 8, 7431-7449, doi:10.5194/acp-8-7431-2008, 2008.

Connolly, P. J., Möhler, O., Field, P. R., Saathoff, H., Burgess, R., Choularton, T., and Gallagher, M.: Studies of heterogeneous freezing by three different desert dust samples, Atmos. Chem. Phys., 9, 2805-2824, doi:10.5194/acp-9-2805-2009, 2009.

Croteau, T., Bertram, A. K., and Patey, G. N.: Observations of highdensity ferroelectric ordered water in kaolinite trenches using Monte Carlo simulations, J. Phys. Chem. A, 114, 8396-8405, 2010 .
DeMott, P. J., Prenni, A. J., Liu, X., Kreidenweis, S. M., Petters, M. D., Twohy, C. H., Richardson, M. S., Eidhammer, T., and Rogers, D. C.: Predicting global atmospheric ice nuclei distributions and their impacts on climate, P. Natl. Acad. Sci. USA, 107, 11217-11222, 2010.

Eadie, W. J.: A molecular Theory of the Homogeneous Nucleation of Ice from Supercooled Water, PhD thesis, University of Chicago, Chicago, USA, 1971.

Edwards, G. R. and Evans, L. F.: Effect of surface charge on ice nucleation by silver iodide, Trans. Faraday Soc., 58, 1649-1655, 1962.

Eidhammer, T., DeMott, P. J., and Kreidenweis, S. M.: A comparison of heterogeneous ice nucleation parameterizations using a parcel model framework, J. Geophys. Res.-Atmos., 114, D06202, doi:10.1029/2008JD011095, 2009.

Ervens, B. and Feingold, G.: On the representation of immersion and condensation freezing in cloud models using different nucleation schemes, Atmos. Chem. Phys., 12, 5807-5826, doi:10.5194/acp-12-5807-2012, 2012.

Fletcher, N. H.: Size Effect in Heterogeneous Nucleation, J. Chem. Phys., 29, 572-576, doi:10.1063/1.1744540, 1958.

Hande, L. B., Engler, C., Hoose, C., and Tegen, I.: Seasonal variability of Saharan desert dust and ice nucleating particles over Europe, Atmos. Chem. Phys., 15, 4389-4397, doi:10.5194/acp15-4389-2015, 2015.

Hiranuma, N., Augustin-Bauditz, S., Bingemer, H., Budke, C., Curtius, J., Danielczok, A., Diehl, K., Dreischmeier, K., Ebert, M., Frank, F., Hoffmann, N., Kandler, K., Kiselev, A., Koop, T., Leisner, T., Möhler, O., Nillius, B., Peckhaus, A., Rose, D., Weinbruch, S., Wex, H., Boose, Y., DeMott, P. J., Hader, J. D., Hill, T. C. J., Kanji, Z. A., Kulkarni, G., Levin, E. J. T., McCluskey, C. S., Murakami, M., Murray, B. J., Niedermeier, D., Petters, M. D., O’Sullivan, D., Saito, A., Schill, G. P., Tajiri, T., Tolbert, M. A., Welti, A., Whale, T. F., Wright, T. P., and Yamashita, K.: A comprehensive laboratory study on the immersion freezing behavior of illite NX particles: a comparison of 17 ice nucleation measurement techniques, Atmos. Chem. Phys., 15, 2489-2518, doi:10.5194/acp-15-2489-2015, 2015.

Hoffmann, N., Duft, D., Kiselev, A., and Leisner, T.: Contact freezing efficiency of mineral dust aerosols studied in an electrodynamic balance: quantitative size and temperature dependence for illite particles, Faraday Discuss., 165, 383-390, 2013.

Hoose, C., Kristjánsson, J., Chen, J.-P., and Hazra, A.: A ClassicalTheory-Based Parameterization of Heterogeneous Ice Nucleation by Mineral Dust, Soot, and Biological Particles in a Global Climate Model, J. Atmos. Sci., 67, 2483-2503, 2010.

$\mathrm{Hu}, \mathrm{X}$. L. and Michaelides, A.: Ice formation on kaolinite: Lattice match or amphoterism?, Surf. Sci., 601, 5378-5381, 2007.

$\mathrm{Hu}$, X. L. and Michaelides, A.: Water on the hydroxylated (001) surface of kaolinite: From monomer adsorption to a flat 2D wetting layer, Surf. Sci., 602, 960-974, 2008.

Hurrell, J., Meehl, G. A., Bader, D., Delworth, T. L., Kirtman, B., and Wielicki, B.: A Unified Modeling Approach to Climate System Prediction, B. Am. Meteorol. Soc., 90, 1819-1832, doi:10.1175/2009BAMS2752.1, 2009.

Ickes, L., Welti, A., Hoose, C., and Lohmann, U.: Classical nucleation theory of homogeneous freezing of water: thermodynamic and kinetic parameters, Phys. Chem. Chem. Phys., 17, 5514 5537, doi:10.1039/C4CP04184D, 2015. 
Kanji, Z. A., Welti, A., Chou, C., Stetzer, O., and Lohmann, U.: Laboratory studies of immersion and deposition mode ice nucleation of ozone aged mineral dust particles, Atmos. Chem. Phys., 13, 9097-9118, doi:10.5194/acp-13-9097-2013, 2013.

Khvorostyanov, V. I. and Curry, J. A.: A new theory of heterogeneous ice nucleation for application in cloud and climate models, Geophys. Res. Lett., 27, 4081-4084, doi:10.1029/1999GL011211, 2000.

Khvorostyanov, V. I. and Curry, J. A.: The Theory of Ice Nucleation by Heterogeneous Freezing of Deliquescent Mixed CCN. Part I: Critical Radius, Energy, and Nucleation Rate, J. Atmos. Sci., 61, 2676-2691, doi:10.1175/JAS3266.1, 2004.

Khvorostyanov, V. I. and Curry, J. A.: The Theory of Ice Nucleation by Heterogeneous Freezing of Deliquescent Mixed CCN. Part II: Parcel Model Simulation, J. Atmos. Sci., 62, 261-285, doi:10.1175/JAS-3367.1, 2005.

Knopf, D. A. and Forrester, S. M.: Freezing of Water and Aqueous $\mathrm{NaCl}$ Droplets Coated by Organic Monolayers as a Function of Surfactant Properties and Water Activity, J. Phys. Chem. A, 115, 5579-5591, doi:10.1021/jp2014644, 2011.

Liu, X., Penner, J. E., Ghan, S. J., and Wang, M.: Inclusion of Ice Microphysics in the NCAR Community Atmospheric Model Version 3 (CAM3), J. Climate, 20, 4526-4547, doi:10.1175/JCLI4264.1, 2007.

Lohmann, U.: A glaciation indirect aerosol effect caused by soot aerosols, Geophys. Res. Lett., 29, 11-1-11-4, doi:10.1029/2001GL014357, 2002.

Lüönd, F., Stetzer, O., Welti, A., and Lohmann, U.: Experimental study on the ice nucleation ability of size-selected kaolinite particles in the immersion mode, J. Geophys. Res.-Atmos., 115, D14201, doi:10.1029/2009JD012959, 2010.

Marcolli, C., Gedamke, S., and Zobrist, B.: Efficiency of Immersion Mode Ice Nucleation on Surrogates of Mineral Dust, in: Nucleation and Atmospheric Aerosols, edited by: O'Dowd, C. D. and Wagner, P. E., 36-40, Springer Netherlands, 2007.

Meyers, M. P., DeMott, P. J., and Cotton, W. R.: New Primary Ice-Nucleation Parameterizations in an Explicit Cloud Model, J. Appl. Meteor., 31, 708-721, doi:10.1175/15200450(1992)031<0708:NPINPI>2.0.CO;2, 1992.

Murray, B. J., Broadley, S. L., Wilson, T. W., Atkinson, J. D., and Wills, R. H.: Heterogeneous freezing of water droplets containing kaolinite particles, Atmos. Chem. Phys., 11, 4191-4207, doi:10.5194/acp-11-4191-2011, 2011.

Niedermeier, D., Shaw, R. A., Hartmann, S., Wex, H., Clauss, T., Voigtländer, J., and Stratmann, F.: Heterogeneous ice nucleation: exploring the transition from stochastic to singular freezing behavior, Atmos. Chem. Phys., 11, 8767-8775, doi:10.5194/acp11-8767-2011, 2011.

Niemand, M., Möhler, O., Vogel, B., Vogel, H., Hoose, C., Connolly, P., Klein, H., Bingemer, H., DeMott, P., Skrotzki, J., and Leisner, T.: A Particle-Surface-Area-Based Parameterization of Immersion Freezing on Desert Dust Particles, J. Atmos. Sci., 69, 3077-3092, doi:10.1175/JAS-D-11-0249.1, 2012.

O’Sullivan, D., Murray, B. J., Malkin, T. L., Whale, T. F., Umo, N. S., Atkinson, J. D., Price, H. C., Baustian, K. J., Browse, J., and Webb, M. E.: Ice nucleation by fertile soil dusts: relative importance of mineral and biogenic components, Atmos. Chem. Phys., 14, 1853-1867, doi:10.5194/acp-14-1853-2014, 2014.
Ouchi, K.: Freezing Mechanism of Supercooled Water, Sci. Rep. Tohoku Univ., 6, 43-61, 1954.

Paukert, M. and Hoose, C.: Modeling immersion freezing with aerosol-dependent prognostic ice nuclei in Arctic mixedphase clouds, J. Geophys. Res.-Atmos., 119, 9073-9092, doi:10.1002/2014JD021917, 2014.

Prenni, A. J., Harrington, J. Y., Tjernström, M., DeMott, P. J., Avramov, A., Long, C. N., Kreidenweis, S. M., Olsson, P. Q., and Verlinde, J.: Can Ice-Nucleating Aerosols Affect Arctic Seasonal Climate?, B. Am. Meteorol. Soc., 88, 541-550, 2007.

Pruppacher, H. R. and Klett, J. D.: Microphysics of Clouds and Precipitation, vol. 18, Kluwer Academic Publishers, 2 edn., Atmospheric and oceanographic sciences library; 2nd rev. and enl. ed., with an Introduction to cloud chemistry and cloud electricity, Dordrecht, the Netherlands, doi:10.1007/978-0-306-48100$0,2000$.

Reinhardt, A. and Doye, J. P. K.: Note: Homogeneous TIP4P/2005 ice nucleation at low supercooling, J. Chem. Phys., 139, 096102, doi:10.1063/1.4819898, 2013.

Rigg, Y. J., Alpert, P. A., and Knopf, D. A.: Immersion freezing of water and aqueous ammonium sulfate droplets initiated by humic-like substances as a function of water activity, Atmos. Chem. Phys., 13, 6603-6622, doi:10.5194/acp-13-6603-2013, 2013.

Šolc, R., Gerzabek, M. H., Lischka, H., and Tunega, D.: Wettability of kaolinite (001) surfaces - Molecular dynamic study, Geoderma, 169, 47-54, 2011.

Storelvmo, T., Hoose, C., and Eriksson, P.: Global modeling of mixed-phase clouds: The albedo and lifetime effects of aerosols, J. Geophys. Res.-Atmos., 116, D05207, doi:10.1029/2010JD014724, 2011.

Tobo, Y., DeMott, P. J., Hill, T. C. J., Prenni, A. J., SwobodaColberg, N. G., Franc, G. D., and Kreidenweis, S. M.: Organic matter matters for ice nuclei of agricultural soil origin, Atmos. Chem. Phys., 14, 8521-8531, doi:10.5194/acp-14-8521-2014, 2014.

Umo, N. S., Murray, B. J., Baeza-Romero, M. T., Jones, J. M., LeaLangton, A. R., Malkin, T. L., O'Sullivan, D., Neve, L., Plane, J. M. C., and Williams, A.: Ice nucleation by combustion ash particles at conditions relevant to mixed-phase clouds, Atmos. Chem. Phys., 15, 5195-5210, doi:10.5194/acp-15-5195-2015, 2015.

Vali, G.: Nucleation terminology, B. Am. Meteorol. Soc., 66, 14261427, 1985.

Volmer, M.: Kinetik der Phasenbildung, in: Die chemische Reaktion, no. 5, Steinkopff-Verlag, Dresden, Germany, 1939.

Wang, Y., Liu, X., Hoose, C., and Wang, B.: Different contact angle distributions for heterogeneous ice nucleation in the Community Atmospheric Model version 5, Atmos. Chem. Phys., 14, 1041110430, doi:10.5194/acp-14-10411-2014, 2014.

Welti, A.: Immersion freezing dataset of kaolinite, illite, montmorillonite, microcline and Arizona test dust (ATD), ETH Zürich, IAC, doi:10.5905/ethz-1007-76, 2015.

Welti, A., Lüönd, F., Kanji, Z. A., Stetzer, O., and Lohmann, U.: Time dependence of immersion freezing: an experimental study on size selected kaolinite particles, Atmos. Chem. Phys., 12, 9893-9907, doi:10.5194/acp-12-9893-2012, 2012.

Wheeler, M. J., Mason, R. H., Steunenberg, K., Wagstaff, M., Chou, C., and Bertram, A. K.: Immersion Freezing of Supermicron Mineral Dust Particles: Freezing Results, Testing Dif- 
ferent Schemes for Describing Ice Nucleation, and Ice Nucleation Active Site Densities, J. Phys. Chem. A, 119, 4358-4372, doi:10.1021/jp507875q, 2014.

Wiacek, A., Peter, T., and Lohmann, U.: The potential influence of Asian and African mineral dust on ice, mixed-phase and liquid water clouds, Atmos. Chem. Phys., 10, 8649-8667, doi:10.5194/acp-10-8649-2010, 2010.

Young, K. C.: The role of contact nucleation in ice phase initiation in clouds, J. Atmos. Sci., 31, 768-776, 1974.
Young, T.: An essay on the cohesion of fluids, Philos. T. R. Soc., 95, 65-87, 1805.

Zeng, X., Tao, W.-K., Zhang, M., Hou, A. Y., Xie, S., Lang, S., Li, X., Starr, D. O., and Li, X.: A contribution by ice nuclei to global warming, Q. J. Roy. Meteor. Soc., 135, 1614-1629, 2009.

Zobrist, B., Koop, T., Luo, B. P., Marcolli, C., and Peter, T.: Heterogeneous Ice Nucleation Rate Coefficient of Water Droplets Coated by a Nonadecanol Monolayer, J. Phys. Chem. C, 111, 2149-2155, doi:10.1021/jp066080w, 2007. 